\title{
Potential Anticancer Agents: Design, Synthesis of New Pyrido[1,2-a] benzimidazoles and Related Derivatives Linked to Alkylating Fragments
}

Salma AZ Darwish ${ }^{1}$, Rasha Y Elbayaa ${ }^{1,2 *}$, Hayam MA Ashour ${ }^{1}$, Mounir A Khalil ${ }^{1}$ and Elsayed AM Badawey ${ }^{1}$

${ }^{1}$ Department of Pharmaceutical Chemistry, Faculty of Pharmacy, Alexandria University, Alexandria 21521, Egypt

${ }^{2}$ Department of Pharmaceutical Chemistry, Faculty of Pharmacy and Drug Manufacturing, Pharos University in Alexandria, 21311, Egypt

\begin{abstract}
The incentive of the present work has been primarily directed towards the design and synthesis of some novel pyrido[1,2-a]benzimidazoles with specific functionalities believed to have alkylation ability. This combination of pharmacological agents may enable synergistic anticancer effect. Nine compounds 5b, 13a, 13d, 13e, 14b, 14c, 15, 16, and 17 were selected by the National Cancer Institute $(\mathrm{NCl})$, Bethseda, Maryland, USA to be evaluated for their in vitro antitumor activity. All the selected compounds were tested initially at a single dose $(10 \mu \mathrm{M})$ in the full $\mathrm{NCl} 60$ cell panel including leukemia, non-small cell lung, colon, CNS, melanoma, ovarian, renal, prostate and breast cancer cell lines. Majority of the test compounds exhibited moderate cytotoxic activity. The highest activity in all the investigated cancer cells was displayed by $14 \mathrm{c}$ against melanoma SK-MEL-5 cell line. This may be due to the impact of the lipophilic trifluoromethyl substitution on the biological activity profile.
\end{abstract}

Keywords: Design; Synthesis; Substituted pyridine; 2-pyridone; Antitumor activity

\section{Introduction}

Cancer is a devastating affliction, the frequency of which is progressively increasing all over the world. Its occurrence is escalating rapidly and is a major cause in health complications [1]. The treatment approach dictates that the treatment of cancer is directed toward eradication of all cancer cells and this is attained by frenziedly discovery of new candidates of anticancer activity [2].

Previously, we have utilized pyrido[1,2-a]benzimidazole (PBI) as a privileged scaffold for the design of many PBI derivatives of potential cytotoxic activity [3-8]. In fact, this ring system is characterized by the presence of pyridine or 2-pyridone units which constitute a subject of great interest due to their extensive presence in the skeletal backbone of many biologically active compounds. They possess a wide variety of biological activities such as antiulcer [9], antidiabetic [9], antiinflammatory [10], anticoagulant [11], antiviral [12], antibacterial [13], antifungal [14] and anticancer activities [15]. Pyridine moiety is one of the building units of some tyrosine-kinase inhibitors; imatinib is used in the treatment of multiple cancers; whereas, sorafenib is used in the treatment of advanced renal and hepatocellular carcinoma [16]. The 2-pyridone unit is an integral part of some cytotoxic agents such as roquinimex which investigated as adjuvant therapy after bone marrow transplantation in chronic myelogenous leukemia [17] and diazaquinomycin $\mathrm{A}$ which demonstrates in vitro cytotoxicity against some tumor cell lines [18].

Among the investigated PBI series,NSC649900 [3], NSC682011 [4] and NSC699944 [5] (Figure 1) were identified by the NCI as promising candidates for further testing in an in vivo anticancer hollow fiber assay because of their good cytotoxic activity and subpanel disease selectivity especially against leukemic cell in the in vitro screen. In fact, the PBI backbone of these compounds demonstrates structural complementarity with the isosteric $\beta$-carboline and pyrido[2,3-a] indolizine (PI) which constitute the key scaffolds of many cytotoxic agents such as the $\beta$-carboline alkaloid harmine which is identified as a useful inhibitor of tumor development [19] and the antitumor antibiotic camptothecin [20].

Biochemical data suggests that camptothecin act as DNA topoisomerase I inhibitor. It possesses a novel mechanism of action involving the inhibition of DNA relaxation by DNA topoisomerase I, and more specifically the stabilization of a covalent binary complex formed between topoisomerase I and DNA [20]. In addition, it is proposed that the planar nature of camptothecin allows its intercalation between DNA base pairs at the site of single-strand cleavage [21]. For this reason, it may be worthy to study the possible interactions of NSC649900, NSC682011 and NSC699944 with topisomerase-I as a target enzyme because of the evident structural complementarity between PBI scaffold of these agents and pyrido[2,3-a]indolizine (PI) backbone of camptothecin (Figure 2). Docking results revealed that NSC649900, NSC682011 and NSC699944 displayed arene-arene interactions with one or more amino acid residues similar to camptothecin.NSC649900 showed arene-arene interactions with DAC113 and TGPB11 residues, in addition to hydrogen bonding with ArgD364; whereas, NSC682011 displayed arene-arene interactions with TGPB1land ArgD364

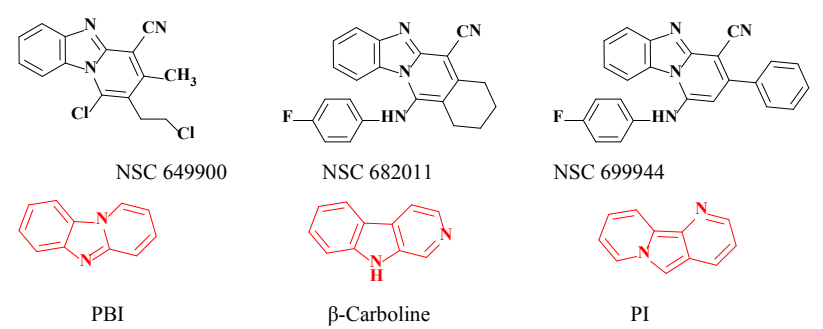

Figure 1: Structural complementarity between PBI, $\beta$-carboline and PI.

*Corresponding author: Rasha $Y$ Elbayaa, Department of Pharmaceutica Chemistry, Faculty of Pharmacy, University of Alexandria, 1Elkhartoum square, Azarita, Alexandria, Egypt, Tel: 002034871317; E-mail: rashabayaa72@gmail.com Received March 09, 2018; Accepted March 15, 2018; Published April 12, 2018

Citation: Darwish SAZ, Elbayaa RY, Ashour HMA, Khalil MA, Badawey EAM (2018) Potential Anticancer Agents: Design, Synthesis of New Pyrido[1,2-a] benzimidazoles and Related Derivatives Linked to Alkylating Fragments. Med Chem (Los Angeles) 8: 86-95. doi: 10.4172/2161-0444.1000498

Copyright: (c) 2018 Darwish SAZ, et al. This is an open-access article distributed under the terms of the Creative Commons Attribution License, which permits unrestricted use, distribution, and reproduction in any medium, provided the original author and source are credited. 
Citation: Darwish SAZ, Elbayaa RY, Ashour HMA, Khalil MA, Badawey EAM (2018) Potential Anticancer Agents: Design, Synthesis of New Pyrido[1,2-a]benzimidazoles and Related Derivatives Linked to Alkylating Fragments. Med Chem (Los Angeles) 8: 86-095. doi: 10.4172/2161. 0444.1000498
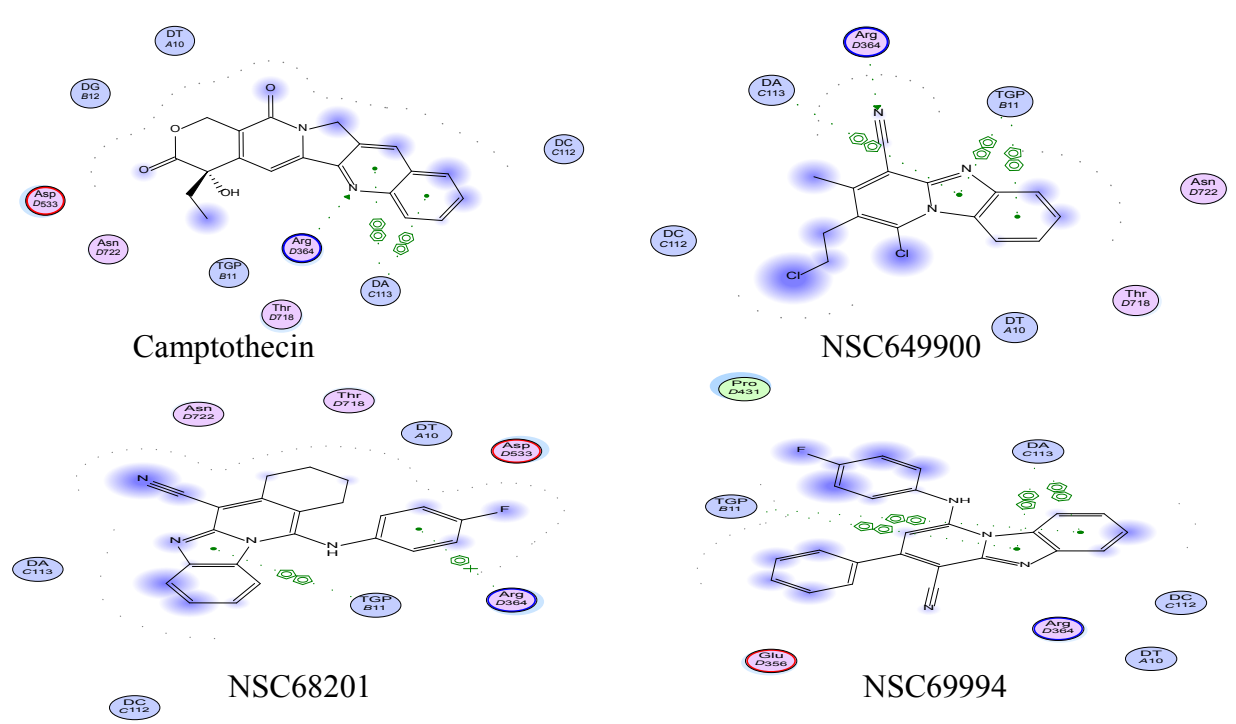

Figure 2: Binding mode of Camptothecin, NSC649900, NSC682011 and NSC699944 into the active sites of topo I-DNA complex (Crystal structure; PDB ID: 1T8I, was obtained from the Protein Data Bank). Docking studies was performed using (MOE 2008.10).

amino acid residues. In addition, NSC699944 revealed arene-arene interactions with TGPB11 and DAC113 amino acid residues. The result indicates that the planar PBI scaffold contribute to binding to the main active sites similar to camptothecin and it is possible that these PBIs may intercalate between DNA base pairs of topo I- DNA complex through arene-arene interactions (Figure 2). Inspired by these findings and in a continuation of our efforts to discover and explore new heterocyclic compounds of promising anticancer activities two series of PBIs comprising pyridine and 2-pyridone nuclei (Figure 3 ) were designed to be synthesized and evaluated for their in vitro anticancer activity. Both series are designed with specific functionalities such as 2-hydoxyalkyl, 2-chloroalkyl, piperidino- and morpholino alkyl moieties at postion-2 through an aminomethylene spacer (Figure 3, Scheme 1) or at position1(Figure 3, Scheme 2). Other analogues comprising aliphatic amino and aryl amino moieties are proposed.

Alkylating fragments such as 2-chloroethylamino and N,N-bis(2-choroethyl)amino and selected substituents such as 2-hydoxyethylamino and morpholinoalkyl are incorporated in the PBI scaffold of the proposed compounds because of their importance in the backbone structure of some antineoplastic drugs; namely, Mitoxantrone [22], Lomustine [23], Bendamustine [24] and Gefitinib [25] .

\section{Experimental}

\section{Chemistry}

All reagents and solvents were purchased from commercial suppliers and were purified and dried when necessary by standard techniques. Melting points were determined in open glass capillaries using Stuart capillary melting point apparatus (Stuart Scientific Stone, Staffordshire, UK) and are uncorrected. IR spectra were recorded, for potassium bromide discs, $\dot{v}\left(\mathrm{~cm}^{-1}\right)$, on Perkin Elmer 1430 spectrophotometer. ${ }^{1} \mathrm{H}-\mathrm{NMR}$ spectra were determined either on a Bruker Avance spectrometer $(400 \mathrm{MHz})$ at the microanalytical unit, Faculty of Science, Cairo University, or on Jeol $(125 \mathrm{MHz})$ at the microanalytical unit, Faculty of Science, Alexandria University, using DMSO- $\mathrm{d}_{6}$ as a solvent and TMS as internal standard. The chemical shifts are given in $\delta \mathrm{ppm}$ values ( $\mathrm{s}$, singlet; $\mathrm{d}$, doublet; $\mathrm{t}$, triplet and

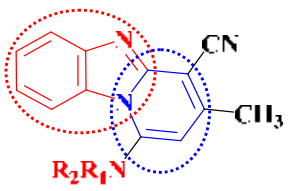

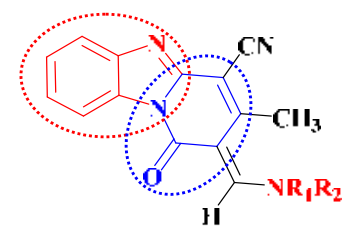<smiles>CCCCC(C)C</smiles><smiles>CCCCCCCC(C)CC(C)CN</smiles>

$(n)$

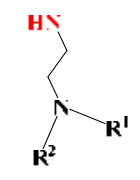

(i.)

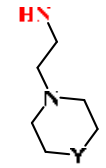

(F')

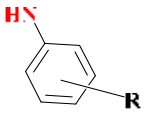

(i)
Figure 3: A display of the proposed PBls and the adopted alkylating fragments and other selected substituents.

m, multiplet). ${ }^{13} \mathrm{C}-\mathrm{NMR}$ spectra were determined on Jeol (125 MHz), Faculty of Science, Alexandria University, using TMS as internal standard. Mass spectra were run on a Finnigan mass spectrometer model SSQ/7000 (70 eV), Faculty of Science, Cairo University. Microanalyses were performed at the microanalytical unit, Faculty of Science, Cairo University. The results of the microanalyses were within $\pm 0.4 \%$ of the calculated values. Follow-up of the reactions and checking the homogeneity of the compounds were made by ascending TLC run on silica gel G (Merck 60) coated glass plates. The spots were visualized by exposure to iodine vapor or UV lamp at $\lambda 254 \mathrm{~nm}$ for few seconds.

Pyridobenzimidazole-4-carbonitrile was prepared according to a reported procedure in a good yield through cyclocondensation of 1H-benzimidazol-2-yl-acetonitrile with ethyl acetoacetate in presence of ammonium acetate [7]. Formylation of pyridobenzimidazole-4- 

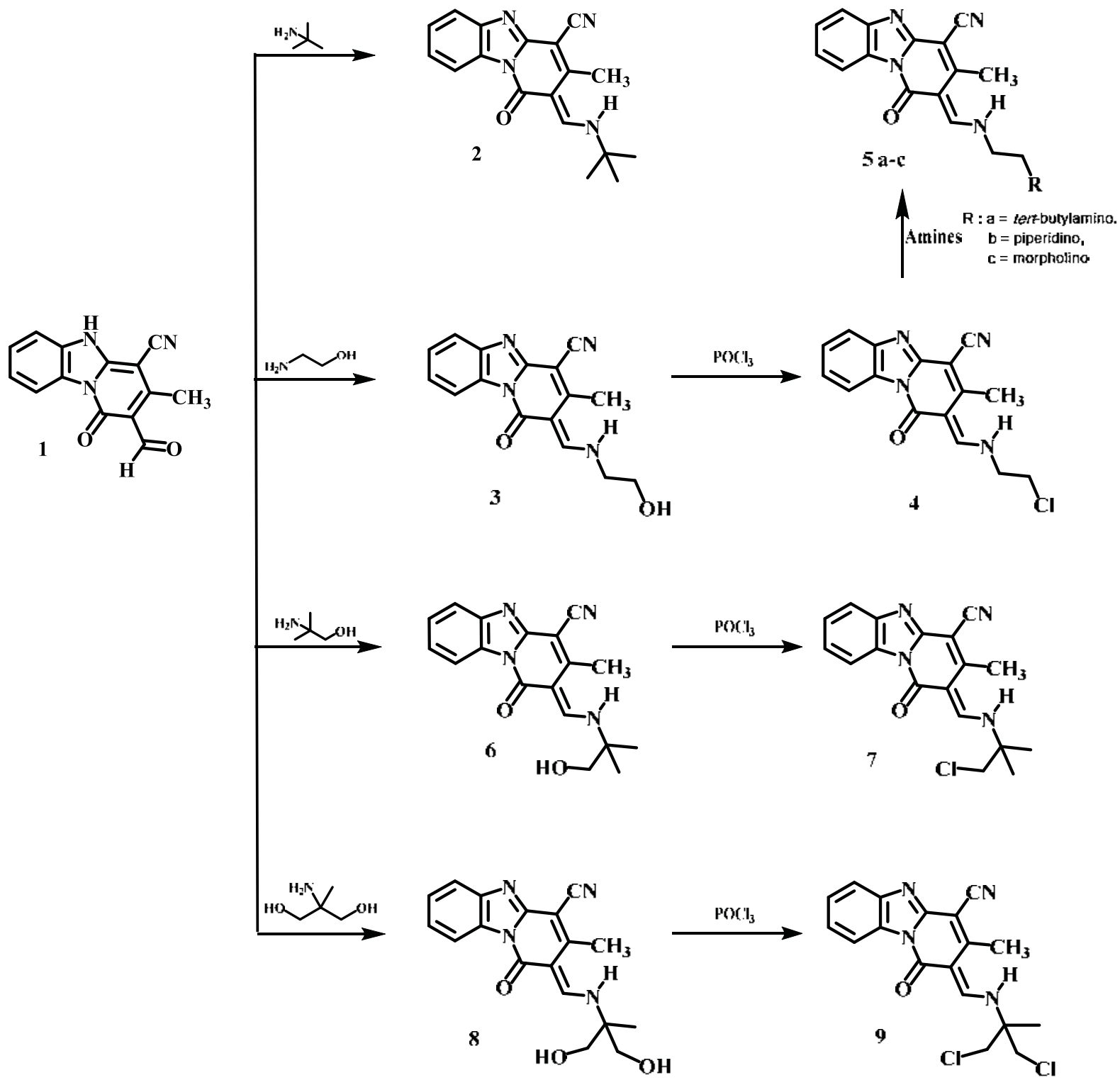

Scheme 1: Synthesis of pyridobenzimidazole derivatives 2-9.

carbonitrile was achieved by applying Vilsmeier Haack reaction by the addition of phosphorous oxychloride to a well stirred suspension of pyridobenzimidazole-4-carbonitrile to afford the aldehyde (1) [7], while 1-Chloro-3-methylpyridobenzimidazole-4-carbonitrile (10) was prepared by refluxing pyridobenzimidazole-4-carbonitrile in excess phosphorous oxychloride according to a reported procedure [7].

2-(tert-Butylamino) methylene-3-methyl-1-oxo-1,2dihydropyrido[1,2-a]benzimidazole-4-carbonitrile (2): A mixture of 2-formyl-3-methyl-1-oxo-1,5-dihydropyridobenzimidazole-4carbonitrile (1) (2 mmol, $0.50 \mathrm{~g}$ ) and 2-methylpropan-2-amine (3 mmol, $0.22 \mathrm{~g})$ in dimethylformamide $(10 \mathrm{ml})$ was stirred at room temperature for $20 \mathrm{~h}$. The reaction mixture was then diluted with ice cold water. The obtained product was filtered, washed with water, dried and crystallized from dimethylformamide/ethanol. Yield 69.63\%, M.P. $>300^{\circ} \mathrm{C}$; IR $\left(\mathrm{KBr}, \mathrm{cm}^{-1}\right): 3231(\mathrm{NH}), 3107,3055,3024,2968,2930$,
$2873(\mathrm{C}-\mathrm{H}), 2218(\mathrm{C} \equiv \mathrm{N}), 1655(\mathrm{C}=\mathrm{O}), 1614(\mathrm{C}=\mathrm{N}), 1561(\mathrm{C}=\mathrm{C})$. ${ }^{1} \mathrm{H}-\mathrm{NMR}$ (400 MHz,DMSO-d $) \delta(\mathrm{ppm}): 1.50\left(\mathrm{~s}, 9 \mathrm{H}, 3 \mathrm{CH}_{3}\right.$ ), 2.67 (s, $3 \mathrm{H}, \mathrm{CH}_{3}$ at $\left.\mathrm{C}_{3}\right), 7.34\left(\mathrm{t}, \mathrm{J}=7.64 \mathrm{~Hz}, 1 \mathrm{H}\right.$, pyridobenzimidazole $\left.\mathrm{C}_{7}-\mathrm{H}\right)$, $7.44\left(\mathrm{t}, \mathrm{J}=7.64 \mathrm{~Hz}, 1 \mathrm{H}\right.$, pyridobenzimidazole $\left.\mathrm{C}_{8}-\mathrm{H}\right), 7.74(\mathrm{~d}, \mathrm{~J}=7.98 \mathrm{~Hz}$, $1 \mathrm{H}$, pyridobenzimidazole $\left.\mathrm{C}_{6}-\mathrm{H}\right), 8.35(\mathrm{~d}, \mathrm{~J}=14.65 \mathrm{~Hz}, 1 \mathrm{H}$, methine $\mathrm{H})$, $8.41\left(\mathrm{~d}, \mathrm{~J}=7.97 \mathrm{~Hz}, 1 \mathrm{H}\right.$, pyridobenzimidazole $\left.\mathrm{C}_{9}-\mathrm{H}\right), 11.60(\mathrm{~d}, \mathrm{~J}=14.23$ $\mathrm{Hz} 1 \mathrm{H}, \mathrm{NH}, \mathrm{D}_{2} \mathrm{O}$ exchangeable). Anal. Calcd. for $\mathrm{C}_{18} \mathrm{H}_{18} \mathrm{~N}_{4} \mathrm{O}$ (306.37): C, 70.57; H, 5.92; N, 18.29. Found: C, 70.74; H, 5.97; N, 18.47.

2-[(2-Hydroxyethyl)amino] methylene-3-methyl-1-oxo-1,2dihydropyrido[1,2-a]benzimidazole-4-carbonitrile (3): A mixture of 2-formyl-3-methyl-1-oxo-1,5-dihydropyridobenzimidazole-4carbonitrile (1) (2 mmol, $0.50 \mathrm{~g})$ and 2-aminoethan-1-ol (3 mmol, 0.18 $\mathrm{g})$ in dimethylformamide $(10 \mathrm{ml})$ was stirred at $60-80^{\circ} \mathrm{C}$ for $4-6 \mathrm{~h}$. The reaction mixture was then diluted with ice cold water. The obtained product was filtered, washed with water, dried and crystallized from 
Citation: Darwish SAZ, Elbayaa RY, Ashour HMA, Khalil MA, Badawey EAM (2018) Potential Anticancer Agents: Design, Synthesis of New Pyrido[1,2-a]benzimidazoles and Related Derivatives Linked to Alkylating Fragments. Med Chem (Los Angeles) 8: 86-095. doi: 10.4172/21610444.1000498

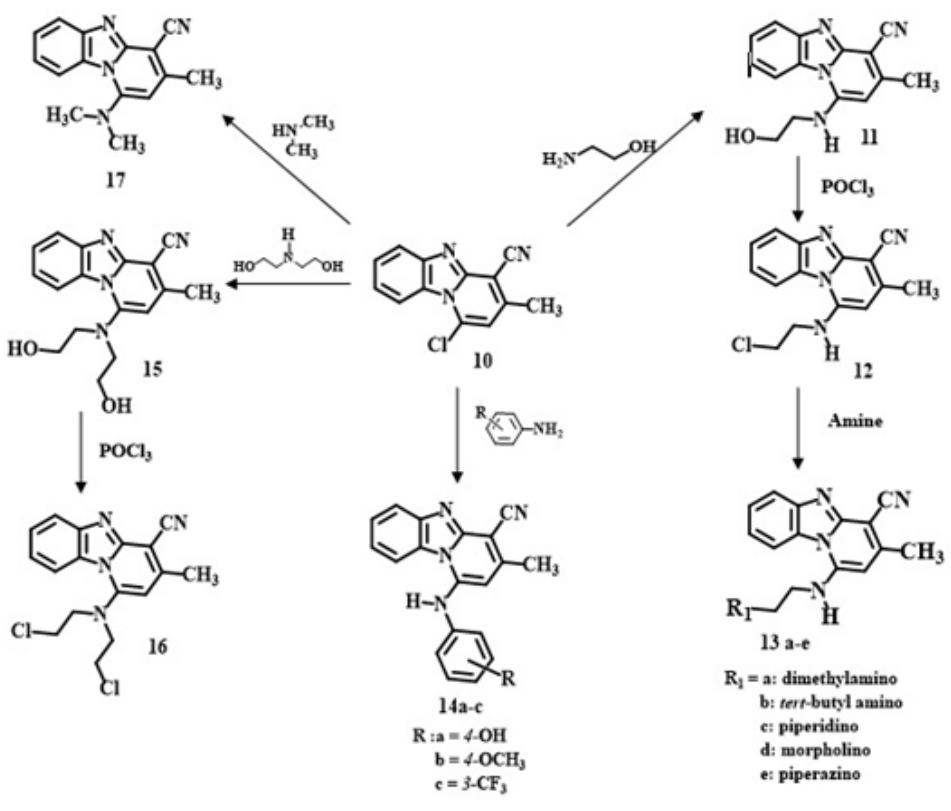

Scheme 2: Synthesis of pyridobenzimidazole derivatives 11-17.

dimethylformamide/ethanol. Yield $50.97 \%$, M.P. $282^{\circ} \mathrm{C}$; IR $(\mathrm{KBr}, \mathrm{cm}$ 1): $3463(\mathrm{OH}), 3250(\mathrm{NH}), 2227(\mathrm{C} \equiv \mathrm{N}), 1649(\mathrm{C}=\mathrm{O}), 1615(\mathrm{C}=\mathrm{N})$, 1562, $1448(\mathrm{C}=\mathrm{C}) .{ }^{1} \mathrm{H}-\mathrm{NMR}\left(400 \mathrm{MHz}, \mathrm{DMSO}-\mathrm{d}_{6}\right) \delta(\mathrm{ppm}): 2.59$ $\left(\mathrm{s}, 3 \mathrm{H}, \mathrm{CH}_{3}\right), 3.69\left(\mathrm{t}, J=7.72 \mathrm{~Hz}, 4 \mathrm{H}, 2 \mathrm{CH}_{2}\right), 5.07(\mathrm{t}, \mathrm{J}=4.53 \mathrm{~Hz}, 1 \mathrm{H}$, $\mathrm{OH}, \mathrm{D}_{2} \mathrm{O}$ exchangeable), $7.33(\mathrm{t}, \mathrm{J}=7.64 \mathrm{~Hz}, 1 \mathrm{H}$, pyridobenzimidazole $\left.\mathrm{C}_{7}-\mathrm{H}\right), 7.44\left(\mathrm{t}, \mathrm{J}=7.64 \mathrm{~Hz}, 1 \mathrm{H}\right.$, pyridobenzimidazole $\left.\mathrm{C}_{8}-\mathrm{H}\right), 7.73(\mathrm{~d}$, $\mathrm{J}=8.02 \mathrm{~Hz}, 1 \mathrm{H}$, pyridobenzimidazole $\left.\mathrm{C}_{6}-\mathrm{H}\right), 8.40(\mathrm{~d}, \mathrm{~J}=8.02 \mathrm{~Hz}, 1 \mathrm{H}$, pyridobenzimidazole $\left.\mathrm{C}_{9}-\mathrm{H}\right), 8.46(\mathrm{~d}, \mathrm{~J}=14.59 \mathrm{~Hz}, 1 \mathrm{H}$, methine $\mathrm{H})$, 11.16-11.33 (m, 1H, NH , $\mathrm{D}_{2} \mathrm{O}$ exchangeable). ${ }^{13} \mathrm{C}-\mathrm{NMR}(125 \mathrm{MHz}$, DMSO-d 6 , $\delta$ ppm): $17.98\left(\mathrm{CH}_{3}\right), 53.12\left(\mathrm{NCH}_{2}\right), 60.14\left(\mathrm{OCH}_{2}\right), 87.10$ (pyridobenzimidazole $\mathrm{C}_{4}$ ), $98.38(\mathrm{CN}), 115.46$ (pyridobenzimidazole $\mathrm{C}_{9}$ ), 116.83 (pyridobenzimidazole $\mathrm{C}_{2}$ ), 119.03, 122.91, 125.63 (pyridobenzimidazole $\mathrm{C}_{6,7.8}$ ), 130.81 (pyridobenzimidazole $\mathrm{C}_{9 \mathrm{a}}$ ), 144.22 (pyridobenzimidazole $\mathrm{C}_{5 \mathrm{a}}$ ), 148.72 (pyridobenzimidazole $\mathrm{C}_{3}$ ), 153.84(pyridobenzimidazole $\mathrm{C}_{4 \mathrm{a}}$ ), 161.56 (methine $\mathrm{CH}$ ), 161.63 (pyridobenzimidazole $\mathrm{C}_{1}$ ). Anal. Calcd. for $\mathrm{C}_{16} \mathrm{H}_{14} \mathrm{~N}_{4} \mathrm{O}_{2}$ (294.31): C, 65.30; H, 4.79; N, 19.04 . Found: C, 65.43; H, 4.85; N, 19.18.

2-[(2-Chloroethyl)amino] methylene-3-methyl-1-oxo-1,2dihydropyrido $[1,2-a]$ benzimidazole-4-carbonitrile (4): A suspension $3(2 \mathrm{mmol}, 0.59 \mathrm{~g})$ in phosphorous oxychloride $(6 \mathrm{ml})$ was heated under reflux for $3 \mathrm{~h}$ while stirring. The reaction mixture was allowed to cool to room temperature and then poured onto crushed ice. The mixture was neutralized with $\mathrm{Na}_{2} \mathrm{CO}_{3}$ and the obtained product was filtered, washed with water, dried and crystallized from DMF/ethanol. Yield 89.53\%, M.P. $>300^{\circ} \mathrm{C}$; IR $\left(\mathrm{KBr}, \mathrm{cm}^{-1}\right): 3231(\mathrm{NH}), 3091,3018,2962$, 2935, $2870(\mathrm{C}-\mathrm{H}), 2214(\mathrm{C} \equiv \mathrm{N}), 1655(\mathrm{C}=\mathrm{O}), 1610(\mathrm{C}=\mathrm{N}), 1556(\mathrm{C}=\mathrm{C})$, 770 (C-Cl). ${ }^{1} \mathrm{H}-\mathrm{NMR}\left(400 \mathrm{MHz}, \mathrm{DMSO}-\mathrm{d}_{6}\right) \delta(\mathrm{ppm}): 2.59$ (s, 3H, $\mathrm{CH}_{3}$ ), 3.91-4.06 (m, $\left.4 \mathrm{H}, 2 \mathrm{CH}_{2}\right), 7.34(\mathrm{t}, \mathrm{J}=7.56 \mathrm{~Hz}, 1 \mathrm{H}$, pyridobenzimidazole $\left.\mathrm{C}_{7}-\mathrm{H}\right), 7.44\left(\mathrm{t}, \mathrm{J}=7.56 \mathrm{~Hz}, 1 \mathrm{H}\right.$, pyridobenzimidazole $\left.\mathrm{C}_{8}-\mathrm{H}\right), 7.73(\mathrm{~d}$, $\mathrm{J}=7.85 \mathrm{~Hz}, 1 \mathrm{H}$, pyridobenzimidazole $\left.\mathrm{C}_{6}-\mathrm{H}\right), 8.38(\mathrm{~d}, \mathrm{~J}=7.83 \mathrm{~Hz}, 1 \mathrm{H}$, pyridobenzimidazole $\left.\mathrm{C}_{9}-\mathrm{H}\right), 8.52(\mathrm{~d}, \mathrm{~J}=14.32 \mathrm{~Hz}, 1 \mathrm{H}$, methine $\mathrm{H})$, 11.11-11.35 (m, 1H, NH, $\mathrm{D}_{2} \mathrm{O}$ exchangeable) ${ }^{13} \mathrm{C}-\mathrm{NMR}(125 \mathrm{MHz}$, DMSO-d,$\delta$ ppm): $18.01\left(\mathrm{CH}_{3}\right), 44.15\left(\mathrm{NCH}_{2}\right), 52.02\left(\mathrm{CH}_{2} \mathrm{Cl}\right), 87.75$ (pyridobenzimidazole $\mathrm{C}_{4}$ ), $98.65(\mathrm{CN}), 115.46$ (pyridobenzimidazole
$\mathrm{C}_{9}$ ), 116.74 (pyridobenzimidazole $\mathrm{C}_{2}$ ), 118.93(pyridobenzimidazole $\mathrm{C}_{6}$ ), 122.93, 125.48 (pyridobenzimidazole $\mathrm{C}_{78}$ ), 130.79(pyridobenzimidazole $\mathrm{C}_{9_{2}}$ ), 144.18 (pyridobenzimidazole $\mathrm{C}_{5}$ ), 148.51(pyridobenzimidazole $\mathrm{C}_{4 \mathrm{a}}$ ), 153.78 (pyridobenzimidazole $\mathrm{C}_{3}$ ), 161.53(pyridobenzimidazole $\mathrm{C}_{1}$ ), 161.76 (methine $\mathrm{CH}$ ). Anal. Calcd. for $\mathrm{C}_{16} \mathrm{H}_{13} \mathrm{ClN}_{4} \mathrm{O}$ (312.76): C, 61.44; H, 4.19; N, 17.91. Found: C, 61.59; H, 4.24; N, 18.07 .

3-Methyl-1-oxo-2-\{[2-(substituted)ethyl] amino $\}$ methylene1,2-dihydropyrido[1,2-a]benzimidazole-4-carbonitrile (5a-c): A suspension of 2-[(2-chloroethyl)amino]methylene-3-methyl-1-oxo1,2-dihydropyridobenzimidazole-4-carbonitrile (4) (2 mmol, 0.63 g) and the corresponding amine $(6 \mathrm{mmol})$ in a mixture of absolute ethanol $(15 \mathrm{ml})$ and dimethylformamide $(6 \mathrm{ml})$ was stirred at room temperature for $10 \mathrm{~h}$ in case of compound (5a) and was refluxed for $10 \mathrm{~h}$ in case of both compounds $(\mathbf{5 b}, \mathbf{c})$. Crushed ice was added to the reaction mixture and the obtained product was filtered, washed with water, dried and crystallized from the proper solvent.

2-\{[2-(tert-Butylamino)ethyl]amino $\}$ methylene-3-methyl-1oxo-1,2-dihydropyrido[1,2-a] benzimidazole-4-carbonitrile (5a): Yield $51.43 \%$, M.P. $>300^{\circ} \mathrm{C}$; crystallization solvent: dioxane/ethanol; IR (KBr, cm $\left.{ }^{-1}\right): 3419(\mathrm{NH}), 3058,2969,2935(\mathrm{C}-\mathrm{H}), 2218(\mathrm{C} \equiv \mathrm{N}), 1655$ $(\mathrm{C}=\mathrm{O}), 1615(\mathrm{C}=\mathrm{N}), 1560(\mathrm{C}=\mathrm{C}) .{ }^{1} \mathrm{H}-\mathrm{NMR}\left(400 \mathrm{MHz}, \mathrm{DMSO}-\mathrm{d}_{6}\right) \delta$ (ppm): 1.50 (s, 9H, $3 \mathrm{CH}_{3}$ ), 2.50 (under DMSO, 4H, $2 \mathrm{NCH}_{2}$ ), 2.68 (s, $3 \mathrm{H}, \mathrm{CH}_{3}$ at $\left.\mathrm{C}_{3}\right), 7.34\left(\mathrm{t}, \mathrm{J}=7.37 \mathrm{~Hz}, 1 \mathrm{H}\right.$, pyridobenzimidazole $\left.\mathrm{C}_{7}-\mathrm{H}\right)$, $7.45\left(\mathrm{t}, \mathrm{J}=7.37 \mathrm{~Hz}, 1 \mathrm{H}\right.$, pyridobenzimidazole $\left.\mathrm{C}_{8}-\mathrm{H}\right), 7.74(\mathrm{~d}, \mathrm{~J}=7.73 \mathrm{~Hz}$, $1 \mathrm{H}$, pyridobenzimidazole $\left.\mathrm{C}_{6}-\mathrm{H}\right), 8.36(\mathrm{~d}, \mathrm{~J}=14.26 \mathrm{~Hz}, 1 \mathrm{H}$, methine $\mathrm{H})$, $8.42\left(\mathrm{~d}, \mathrm{~J}=7.88 \mathrm{~Hz}, 1 \mathrm{H}\right.$, pyridobenzimidazole $\left.\mathrm{C}_{9}-\mathrm{H}\right), 11.61(\mathrm{~m}, \mathrm{~J}=13.82$ $\mathrm{Hz}, 1 \mathrm{H}$, enamine $\mathrm{NH}, \mathrm{D}_{2} \mathrm{O}$ exchangeable). Anal. Calcd. for $\mathrm{C}_{20} \mathrm{H}_{23} \mathrm{~N}_{5} \mathrm{O}$ (349.44): C, 68.74; H, 6.63; N, 20.04. Found: C, 68.98; H, 6.69; N, 20.21.

3-Methyl-1-oxo-2-\{[2-(piperidin-1-yl)ethyl]amino $\}$ methylene1,2-dihydropyrido[1,2-a]benzimidazole-4-carbonitrile (5b): Yield $42.88 \%$, M.P. $175^{\circ} \mathrm{C}$; crystallization solvent: Dichloro-methane/ethyl acetate; IR $\left(\mathrm{KBr}, \mathrm{cm}^{-1}\right): 3420,3246(\mathrm{NH}), 3050,2919,2848,2802(\mathrm{C}$ $\mathrm{H}), 2213(\mathrm{C} \equiv \mathrm{N}), 1659(\mathrm{C}=\mathrm{O}), 1612(\mathrm{C}=\mathrm{N}), 1552(\mathrm{C}=\mathrm{C}) .{ }^{1} \mathrm{H}-\mathrm{NMR}(400$ 
Citation: Darwish SAZ, Elbayaa RY, Ashour HMA, Khalil MA, Badawey EAM (2018) Potential Anticancer Agents: Design, Synthesis of New Pyrido[1,2-a]benzimidazoles and Related Derivatives Linked to Alkylating Fragments. Med Chem (Los Angeles) 8: 86-095. doi: 10.4172/21610444.1000498

$\mathrm{MHz}$, DMSO-d $\left.\mathrm{d}_{6}\right) \delta(\mathrm{ppm}): 1.40-1.53\left(\mathrm{~m}, 6 \mathrm{H}\right.$, piperidine $\left.\mathrm{C}_{3,4,5}-\mathrm{H}_{2}\right), 2.44-$ $2.50\left(\mathrm{~m}, 6 \mathrm{H}, \mathrm{CH}_{2} \mathrm{~N}\right.$ and piperidine $\left.\mathrm{C}_{2,6}-\mathrm{H}_{2}\right), 2.60\left(\mathrm{~s}, 3 \mathrm{H}, \mathrm{CH}_{3}\right), 3.71$ $\left(\mathrm{m}, 2 \mathrm{H}, \mathrm{CH}_{2}-\mathrm{N}\right), 7.33\left(\mathrm{t}, \mathrm{J}=7.64 \mathrm{~Hz}, 1 \mathrm{H}\right.$, pyridobenzimidazole $\left.\mathrm{C}_{7}-\mathrm{H}\right)$, $7.43\left(\mathrm{t}, \mathrm{J}=7.64 \mathrm{~Hz}, 1 \mathrm{H}\right.$, pyridobenzimidazole $\left.\mathrm{C}_{8}-\mathrm{H}\right), 7.72(\mathrm{~d}, \mathrm{~J}=7.93 \mathrm{~Hz}$, $1 \mathrm{H}$, pyridobenzimidazole $\left.\mathrm{C}_{6}-\mathrm{H}\right), 8.39(\mathrm{~d}, \mathrm{~J}=8.01 \mathrm{~Hz}, 1 \mathrm{H}$, pyridobenzimidazole $\left.\mathrm{C}_{9}-\mathrm{H}\right), 8.46(\mathrm{~d}, \mathrm{~J}=14.32 \mathrm{~Hz}, 1 \mathrm{H}$, methine $\mathrm{H}), 11.18(\mathrm{~m}, 1 \mathrm{H}$, $\mathrm{NH}, \mathrm{D}_{2} \mathrm{O}$ exchangeable). Anal. Calcd. for $\mathrm{C}_{21} \mathrm{H}_{23} \mathrm{~N}_{5} \mathrm{O}$ (361.45): C, 69.78; H, 6.41; N, 19.38. Found: C, 69.93; H, 6.52; N, 19.62 .

3-Methyl-2-\{[2-(morpholin-4-yl)ethyl $]$ amino $\}$ methylene-1oxo-1,2-dihydropyrido[1,2-a] benzimidazole-4-carbonitrile (5c): Yield $39.73 \%$, M.P. $240^{\circ} \mathrm{C}$;crystallization solvent: ethanol; IR $(\mathrm{KBr}$, $\left.\mathrm{cm}^{-1}\right)$ : 3415, $3227(\mathrm{NH}), 3050,2920,2866,2804(\mathrm{C}-\mathrm{H}), 2214(\mathrm{C} \equiv \mathrm{N})$, $1665(\mathrm{C}=\mathrm{O}), 1612(\mathrm{C}=\mathrm{N}), 1559(\mathrm{C}=\mathrm{C}) .{ }^{1} \mathrm{H}-\mathrm{NMR}\left(400 \mathrm{MHz}, \mathrm{DMSO}-\mathrm{d}_{6}\right)$ $\delta(\mathrm{ppm}): 2.42-2.52\left(\mathrm{~m}, 5 \mathrm{H}, \mathrm{CH}_{3}\right.$ and $\left.\mathrm{N}-\mathrm{CH}_{2}\right), 2.54-2.66(\mathrm{~m}, 4 \mathrm{H}$, morpholine $\left.\mathrm{C}_{3,5}-\mathrm{H}_{2}\right)$, 3.55-3.62 $\left(\mathrm{m}, 4 \mathrm{H}\right.$, morpholine $\left.\mathrm{C}_{2,6}-\mathrm{H}_{2}\right)$, 3.68$3.78\left(\mathrm{~m}, 2 \mathrm{H}, \mathrm{CH}_{2}-\mathrm{N}\right), 7.33(\mathrm{t}, \mathrm{J}=7.65 \mathrm{~Hz}, 1 \mathrm{H}$, pyridobenzimidazole $\left.\mathrm{C}_{7}-\mathrm{H}\right), 7.43\left(\mathrm{t}, \mathrm{J}=7.65 \mathrm{~Hz}, 1 \mathrm{H}\right.$, pyridobenzimidazole $\left.\mathrm{C}_{8}-\mathrm{H}\right), 7.72(\mathrm{~d}$, $\mathrm{J}=8.09 \mathrm{~Hz}, 1 \mathrm{H}$, pyridobenzimidazole $\left.\mathrm{C}_{6}-\mathrm{H}\right), 8.39(\mathrm{~d}, \mathrm{~J}=8.10 \mathrm{~Hz}, 1 \mathrm{H}$, pyridobenzimidazole $\left.\mathrm{C}_{9}-\mathrm{H}\right), 8.47(\mathrm{~d}, \mathrm{~J}=14.42 \mathrm{~Hz}, 1 \mathrm{H}$, methine $\mathrm{H})$, $11.20\left(\mathrm{~m}, \mathrm{~J}=13.10 \mathrm{~Hz}, 1 \mathrm{H}, \mathrm{NH}, \mathrm{D}_{2} \mathrm{O}\right.$ exchangeable). Anal. Calcd. for $\mathrm{C}_{20} \mathrm{H}_{21} \mathrm{~N}_{5} \mathrm{O}_{2}$ (363.42): C, 66.10; H, 5.82; N, 19.27. Found: C, 66.37; H, $5.87 ; \mathrm{N}, 19.43$.

2-[(1-Hydroxy-2-methylprop-2-yl)amino]methylene-3-methyl1-oxo-1,2-dihydropyrido[1,2-a]benzimidazole-4-carbonitrile (6): A mixtureof2-formyl-3-methyl-1-oxo-1,5-dihydropyridobenzimidazole4-carbonitrile (1) (2 mmol, $0.50 \mathrm{~g}$ ) and 2-amino-2-methylpropan-1ol $(3 \mathrm{mmol}, 0.27 \mathrm{~g})$ in dimethylformamide $(10 \mathrm{ml})$ was stirred at 60 $80^{\circ} \mathrm{C}$ for $4-6 \mathrm{~h}$. The reaction mixture was then diluted with ice cold water. The obtained product was filtered, washed with water, dried and crystallized from dimethylformamide/ethanol. Yield $83.75 \%$, M.P. $>300^{\circ} \mathrm{C}$; IR $\left(\mathrm{KBr}, \mathrm{cm}^{-1}\right): 3456(\mathrm{OH}), 3280(\mathrm{NH}), 3055,2931,2860$ $(\mathrm{C}-\mathrm{H}), 2218(\mathrm{C} \equiv \mathrm{N}), 1656(\mathrm{C}=\mathrm{O}), 1615(\mathrm{C}=\mathrm{N}), 1561,1446(\mathrm{C}=\mathrm{C}) .{ }^{1} \mathrm{H}-$ NMR (400 MHz,DMSO-d $) \delta(\mathrm{ppm}): 1.42\left(\mathrm{~s}, 6 \mathrm{H}, 2 \mathrm{CH}_{3}\right), 2.64(\mathrm{~s}, 3 \mathrm{H}$, $\mathrm{CH}_{3}$ at $\left.\mathrm{C}_{3}\right), 3.50\left(\mathrm{~d}, \mathrm{~J}=5.16 \mathrm{~Hz}, 2 \mathrm{H}, \mathrm{CH}_{2}-\mathrm{O}\right), 5.53(\mathrm{t}, \mathrm{J}=5.26 \mathrm{~Hz}, 1 \mathrm{H}, \mathrm{OH}$ , $\mathrm{D}_{2} \mathrm{O}$ exchangeable), $7.33\left(\mathrm{t}, \mathrm{J}=7.67 \mathrm{~Hz}, 1 \mathrm{H}\right.$, pyridobenzimidazole $\mathrm{C}_{7}$ $\mathrm{H}), 7.4\left(\mathrm{t}, \mathrm{J}=7.67 \mathrm{~Hz}, 1 \mathrm{H}\right.$, pyridobenzimidazole $\left.\mathrm{C}_{8}-\mathrm{H}\right), 7.72(\mathrm{~d}, \mathrm{~J}=8.03$ $\mathrm{Hz}, 1 \mathrm{H}$, pyridobenzimidazole $\left.\mathrm{C}_{6}-\mathrm{H}\right), 8.31(\mathrm{~d}, \mathrm{~J}=14.71 \mathrm{~Hz}, 1 \mathrm{H}$, methine $\mathrm{H}), 8.39\left(\mathrm{~d}, \mathrm{~J}=7.48 \mathrm{~Hz}, 1 \mathrm{H}\right.$, pyridobenzimidazole $\left.\mathrm{C}_{9}-\mathrm{H}\right), 11.68(\mathrm{~d}$, $\mathrm{J}=14.20 \mathrm{~Hz}, 1 \mathrm{H}, \mathrm{NH}, \mathrm{D}_{2} \mathrm{O}$ exchangeable). Anal. Calcd. for $\mathrm{C}_{18} \mathrm{H}_{18} \mathrm{~N}_{4} \mathrm{O}_{2}$ (322.37): C, 67.07; H, 5.63; N, 17.38. Found: C, 67.21; H, 5.72; N, 17.52.

2-[(1-Chloro-2-methylprop-2-yl)amino] methylene-3-methyl-1oxo-1,2-dihydropyrido[1,2-a] benzimidazole-4-carbonitrile (7): A suspension $6(2 \mathrm{mmol}, 0.64 \mathrm{~g})$ in phosphorous oxychloride $(6 \mathrm{ml})$ was heated under reflux for $3 \mathrm{~h}$ while stirring. The reaction mixture was allowed to cool to room temperature and then poured onto crushed ice. The mixture was neutralized with $\mathrm{Na}_{2} \mathrm{CO}_{3}$ and the obtained product was filtered, washed with water, dried and crystallized from dioxane/ethanol. Yield 58.68\%, M.P. $276^{\circ} \mathrm{C}$; IR $\left(\mathrm{KBr}, \mathrm{cm}^{-1}\right): 3231$ $(\mathrm{NH}), 3054,3022,2977,2929(\mathrm{C}-\mathrm{H}), 2220(\mathrm{C} \equiv \mathrm{N}), 1655(\mathrm{C}=\mathrm{O})$, $1611(\mathrm{C}=\mathrm{N}), 1561,1436(\mathrm{C}=\mathrm{C}) .{ }^{1} \mathrm{H}-\mathrm{NMR}\left(400 \mathrm{MHz}, \mathrm{DMSO}-\mathrm{d}_{6}\right)$ $\delta$ (ppm): $1.57\left(\mathrm{~s}, 6 \mathrm{H}, 2 \mathrm{CH}_{3}\right), 2.66\left(\mathrm{~s}, 3 \mathrm{H}, \mathrm{CH}_{3}\right.$ at $\left.\mathrm{C}_{3}\right), 4.02(\mathrm{~s}, 2 \mathrm{H}$, $\left.\mathrm{CH}_{2}-\mathrm{Cl}\right), 7.34\left(\mathrm{t}, \mathrm{J}=7.74 \mathrm{~Hz}, 1 \mathrm{H}\right.$ pyridobenzimidazole $\left.\mathrm{C}_{7}-\mathrm{H}\right), 7.45(\mathrm{t}$, $\mathrm{J}=7.74 \mathrm{~Hz}, 1 \mathrm{H}$, pyridobenzimidazole $\left.\mathrm{C}_{8}-\mathrm{H}\right), 7.74(\mathrm{~d}, \mathrm{~J}=7.81 \mathrm{~Hz}, 1 \mathrm{H}$, pyridobenzimidazole $\left.\mathrm{C}_{6}-\mathrm{H}\right), 8.32(\mathrm{~d}, \mathrm{~J}=14.29 \mathrm{~Hz}, 1 \mathrm{H}$, methine $\mathrm{H}), 8.40$ (d, J=7.94 Hz, $1 \mathrm{H}$, pyridobenzimidazole $\left.\mathrm{C}_{9}-\mathrm{H}\right), 11.60(\mathrm{~d}, \mathrm{~J}=14.43 \mathrm{~Hz}$, $1 \mathrm{H}, \mathrm{NH}, \mathrm{D}_{2} \mathrm{O}$ exchangeable). Anal. Calcd. for $\mathrm{C}_{18} \mathrm{H}_{17} \mathrm{ClN}_{4} \mathrm{O}$ (340.81): $\mathrm{C}$, 63.44; H, 5.03; N, 16.44 . Found: C, 63.59; H, 5.11; N, 16.72.

2-[(1,3-Dihydroxy-2-methylprop-2-yl)amino] methylene3-methyl-1-oxo-1,2-dihydropyrido[1,2-a]benzimidazole-4- carbonitrile (8): A mixture of 2-formyl-3-methyl-1-oxo-1,5dihydropyridobenzimidazole-4-carbonitrile (1) (2 mmol, 0.50 g) and 2-amino-2-methylpropane-1,3-diol (3 mmol, $0.315 \mathrm{~g})$ in dimethylformamide $(10 \mathrm{ml})$ was stirred at $60-80^{\circ} \mathrm{C}$ for $4-6 \mathrm{~h}$ The reaction mixture was then diluted with ice cold water. The obtained product was filtered, washed with water, dried and crystallized from dimethylformamide/ethanol. Yield $63.54 \%$, M.P. $>300^{\circ} \mathrm{C}$; IR $(\mathrm{KBr}, \mathrm{cm}$ $\left.{ }^{1}\right)$ : 3472, $3361(\mathrm{OH}), 3160(\mathrm{NH}), 2942,2865(\mathrm{C}-\mathrm{H}), 2212(\mathrm{C} \equiv \mathrm{N}), 1661$ $(\mathrm{C}=\mathrm{O}), 1610(\mathrm{C}=\mathrm{N}), 1556,1446(\mathrm{C}=\mathrm{C}) .{ }^{1} \mathrm{H}-\mathrm{NMR}\left(400 \mathrm{MHz}, \mathrm{DMSO}-\mathrm{d}_{6}\right)$ $\delta(\mathrm{ppm}): 1.36\left(\mathrm{~s}, 3 \mathrm{H}, \mathrm{CH}_{3}\right), 2.63\left(\mathrm{~s}, 3 \mathrm{H}, \mathrm{CH}_{3}\right.$ at $\left.\mathrm{C}_{3}\right), 3.51,3.55(2 \mathrm{~d}$, $\mathrm{J}_{1}=5.44 \mathrm{~Hz}, \mathrm{~J}_{2}=5.21 \mathrm{~Hz}$, each $\left.1 \mathrm{H}, \mathrm{CH}_{2}\right), 3.62,3.66\left(2 \mathrm{~d}, \mathrm{~J}_{1}=4.91\right.$ $\mathrm{Hz}, \mathrm{J}_{2}=5.16 \mathrm{~Hz}$, each $\left.1 \mathrm{H}, \mathrm{CH}_{2}\right), 5.41\left(\mathrm{t}, \mathrm{J}=5.08 \mathrm{~Hz}, 2 \mathrm{H}, 2 \mathrm{OH}, \mathrm{D}_{2} \mathrm{O}\right.$ exchangeable), $7.33\left(\mathrm{t}, \mathrm{J}=7.67 \mathrm{~Hz}, 1 \mathrm{H}\right.$, pyridobenzimidazole $\left.\mathrm{C}_{7}-\mathrm{H}\right)$, $7.44\left(\mathrm{t}, \mathrm{J}=7.67 \mathrm{~Hz}, 1 \mathrm{H}\right.$, pyridobenzimidazole $\left.\mathrm{C}_{8}-\mathrm{H}\right), 7.73(\mathrm{~d}, \mathrm{~J}=8.04 \mathrm{~Hz}$, $1 \mathrm{H}$, pyridobenzimidazole $\left.\mathrm{C}_{6}-\mathrm{H}\right), 8.32(\mathrm{~d}, \mathrm{~J}=14.77 \mathrm{~Hz}, 1 \mathrm{H}$, methine $\mathrm{H})$, $8.40\left(\mathrm{~d}, \mathrm{~J}=8.48 \mathrm{~Hz}, 1 \mathrm{H}\right.$, pyridobenzimidazole $\left.\mathrm{C}_{9}-\mathrm{H}\right), 11.70(\mathrm{~d}, \mathrm{~J}=14.64$ $\mathrm{Hz}, 1 \mathrm{H}, \mathrm{NH}, \mathrm{D}_{2} \mathrm{O}$ exchangeable). Anal. Calcd. for $\mathrm{C}_{18} \mathrm{H}_{18} \mathrm{~N}_{4} \mathrm{O}_{3}$ (338.37): C, 63.89; H, 5.36; N, 16.56. Found: C, 64.06; H, 5.40; N, 16.73.

2-[(1,3-Dichloro-2-methylprop-2-yl)amino]methylene3-methyl-1-oxo-1,2-dihydropyrido[1,2-a]benzimidazole-4carbonitrile (9): A suspension 8 (2mmol, $0.68 \mathrm{~g})$ in phosphorous oxychloride $(6 \mathrm{ml})$ was heated under reflux for $3 \mathrm{~h}$ while stirring. The reaction mixture was allowed to cool to room temperature and then poured onto crushed ice. The mixture was neutralized with $\mathrm{Na}_{2} \mathrm{CO}$ and the obtained product was filtered, washed with water, dried and crystallized from absolute ethanol. Yield $15.99 \%$, M.P. $>300^{\circ} \mathrm{C}$; IR $(\mathrm{KBr}$, $\left.\mathrm{cm}^{-1}\right): 3365(\mathrm{NH})$, 3232, $2965(\mathrm{C}-\mathrm{H}), 2218(\mathrm{C} \equiv \mathrm{N}), 1653(\mathrm{C}=\mathrm{O}), 1608$ $(\mathrm{C}=\mathrm{N}), 1559,1444(\mathrm{C}=\mathrm{C}) .{ }^{1} \mathrm{H}-\mathrm{NMR}\left(400 \mathrm{MHz}, \mathrm{DMSO}-\mathrm{d}_{6}\right) \delta(\mathrm{ppm})$ : $1.66\left(\mathrm{~s}, 3 \mathrm{H}, \mathrm{CH}_{3}\right), 2.65\left(\mathrm{~s}, 3 \mathrm{H}, \mathrm{CH}_{3}\right.$ at $\left.\mathrm{C}_{3}\right), 4.10,4.15(2 \mathrm{~s}$, each $2 \mathrm{H}, 2$ $\left.\mathrm{CH}_{2}-\mathrm{Cl}\right), 7.34\left(\mathrm{t}, \mathrm{J}=7.67 \mathrm{~Hz}, 1 \mathrm{H}\right.$, pyridobenzimidazole $\left.\mathrm{C}_{7}-\mathrm{H}\right), 7.43(\mathrm{t}$, $\mathrm{J}=7.67 \mathrm{~Hz}, 1 \mathrm{H}$,pyridobenzimidazole $\left.\mathrm{C}_{8}-\mathrm{H}\right), 7.73(\mathrm{~d}, \mathrm{~J}=8.04 \mathrm{~Hz}, 1 \mathrm{H}$, pyridobenzimidazole $\left.\mathrm{C}_{6}-\mathrm{H}\right), 8.35(\mathrm{~d}, \mathrm{~J}=14.18 \mathrm{~Hz}, 1 \mathrm{H}$, methine $\mathrm{H}), 8.40$ (d, J=8.48 Hz, $1 \mathrm{H}$, pyridobenzimidazole $\left.\mathrm{C}_{9}-\mathrm{H}\right), 11.63(\mathrm{~d}, \mathrm{~J}=12.96 \mathrm{~Hz}$, $1 \mathrm{H}, \mathrm{NH}, \mathrm{D}_{2} \mathrm{O}$ exchangeable). Anal. Calcd. for $\mathrm{C}_{18} \mathrm{H}_{16} \mathrm{Cl}_{2} \mathrm{~N}_{4} \mathrm{O}$ (375.25): C, $57.61 ; \mathrm{H}, 4.30 ; \mathrm{N}, 14.93$. Found: C, 57.84; H, 4.36; N, 15.18 .

1 - ( 2 - Hydroxyethyl) a mino-3 - methylpyrido [ 1,2 - a $]$ benzimidazole-4-carbonitrile (11): A mixture of the chloro derivative (10) $(4 \mathrm{mmol}, 0.97 \mathrm{~g})$ and ethanolamine, (12 mmoles, $0.73 \mathrm{~g})$ in dioxane $(20 \mathrm{ml})$ was heated at $60-80^{\circ} \mathrm{C}$ for $10 \mathrm{~h}$. The reaction mixture was then poured into ice cold water. The product was filtered, washed with water, dried and crystallized from DMF/ ethanol. Yield 93.46\%, M.P. $286^{\circ} \mathrm{C}$; IR $\left(\mathrm{KBr}, \mathrm{cm}^{-1}\right): 3423(\mathrm{OH}), 3256(\mathrm{NH}), 2923,2872(\mathrm{C}$ $\mathrm{H}), 2212(\mathrm{C} \equiv \mathrm{N}), 1630(\mathrm{C}=\mathrm{N}), 1590,1563(\mathrm{C}=\mathrm{C}), 1084\left(\mathrm{CH}_{2}-\mathrm{OH}\right)$. 1H-NMR (400 MHz,DMSO-d $)_{6} \delta(\mathrm{ppm}): 2.51\left(\mathrm{~s}, 3 \mathrm{H}, \mathrm{CH}_{3}\right), 3.55(\mathrm{q}$, $\left.2 \mathrm{H}, \mathrm{J}=5.74 \mathrm{~Hz}, \mathrm{~N}-\mathrm{CH}_{2}\right), 3.76\left(\mathrm{q}, 2 \mathrm{H}, \mathrm{J}=5.73 \mathrm{~Hz}, \mathrm{O}-\mathrm{CH}_{2}\right), 5.01(\mathrm{t}, \mathrm{J}=5.57$, $1 \mathrm{H}, \mathrm{OH}, \mathrm{D} 2 \mathrm{O}$ exchangeable), $6.18\left(\mathrm{~s}, 1 \mathrm{H}\right.$, pyridobenzimidazole $\left.\mathrm{C}_{2}-\mathrm{H}\right)$, $7.24\left(\mathrm{t}, \mathrm{J}=5.43 \mathrm{~Hz}, 1 \mathrm{H}, \mathrm{NH}, \mathrm{D}_{2} \mathrm{O}\right.$ exchangeable), $7.31(\mathrm{t}, \mathrm{J}=7.73 \mathrm{~Hz}, 1 \mathrm{H}$, pyridobenzimidazole $\left.\mathrm{C}_{7}-\mathrm{H}\right), 7.51(\mathrm{t}, \mathrm{J}=7.73 \mathrm{~Hz}, 1 \mathrm{H}$, pyridobenzimidazole $\left.\mathrm{C}_{8}-\mathrm{H}\right), 7.77\left(\mathrm{~d}, \mathrm{~J}=8.14 \mathrm{~Hz}, 1 \mathrm{H}\right.$, pyridobenzimidazole $\left.\mathrm{C}_{6}-\mathrm{H}\right), 8.37(\mathrm{~d}$, $\mathrm{J}=8.37 \mathrm{~Hz}, 1 \mathrm{H}$, pyridobenzimidazole $\left.\mathrm{C}_{9}-\mathrm{H}\right)$. Anal. Calcd. for $\mathrm{C}_{15} \mathrm{H}_{14} \mathrm{~N}_{4} \mathrm{O}$ (266.30): C, 67.65; H, 5.30; N, 21.04. Found: C, 67.89; H, 5.39; N, 21.25.

1-(2-Chloroethyl)amino-3-methylpyrido[1,2-a]benzimidazole4-carbonitrile (12): A suspension of 1-(2-hydroxyethyl)amino-3methylpyrido[1,2-a]benzimidazole-4-carbonitrile (11) (5 mmol, 1.3 g) in phosphorous oxychloride $(5 \mathrm{ml})$ was heated under reflux while stirring for $3 \mathrm{~h}$. The reaction mixture was allowed to cool to room temperature, then poured onto crushed ice and neutralized with $\mathrm{Na}_{2} \mathrm{CO}_{3}$. The product was filtered, washed with water and crystallized from dioxane. Yield 17.56\%, M.P. $244^{\circ} \mathrm{C}$; IR $\left(\mathrm{KBr}, \mathrm{cm}^{-1}\right): 3413(\mathrm{NH})$, 3104, 3069, 3023, 2966, $2921(\mathrm{C}-\mathrm{H}), 2211(\mathrm{C} \equiv \mathrm{N}), 1630(\mathrm{C}=\mathrm{N}), 1600$ 
Citation: Darwish SAZ, Elbayaa RY, Ashour HMA, Khalil MA, Badawey EAM (2018) Potential Anticancer Agents: Design, Synthesis of New Pyrido[1,2-a]benzimidazoles and Related Derivatives Linked to Alkylating Fragments. Med Chem (Los Angeles) 8: 86-095. doi: 10.4172/21610444.1000498

1552, 1489, $1462(\mathrm{C}=\mathrm{C}) .{ }^{1} \mathrm{H}-\mathrm{NMR}\left(400 \mathrm{MHz}, \mathrm{DMSO}-\mathrm{d}_{6}\right) \delta(\mathrm{ppm})$ : $2.54\left(\mathrm{~s}, 3 \mathrm{H}, \mathrm{CH}_{3}\right), 3.85\left(\mathrm{q}, \mathrm{J}=5.67 \mathrm{~Hz}, 2 \mathrm{H}, \mathrm{N}-\mathrm{CH}_{2}\right), 3.98(\mathrm{t}, \mathrm{J}=6.14$ $\left.\mathrm{Hz}, 2 \mathrm{H}, \mathrm{CH}_{2}-\mathrm{Cl}\right), 6.30\left(\mathrm{~s}, 1 \mathrm{H}\right.$, pyridobenzimidazole $\left.\mathrm{C}_{2}-\mathrm{H}\right), 7.35$ $\left(\mathrm{t}, \mathrm{J}=7.81 \mathrm{~Hz}, 1 \mathrm{H}\right.$, pyridobenzimidazole $\left.\mathrm{C}_{7}-\mathrm{H}\right), 7.49-7.58(\mathrm{~m}, 2 \mathrm{H}$, pyridobenzimidazole $\mathrm{C}_{8}-\mathrm{H}$ and $\mathrm{NH}, \mathrm{D}_{2} \mathrm{O}$ exchangeable), $7.79(\mathrm{~d}$, $\mathrm{J}=8.16 \mathrm{~Hz}, 1 \mathrm{H}$, pyridobenzimidazole $\left.\mathrm{C}_{6}-\mathrm{H}\right), 8.42(\mathrm{~d}, \mathrm{~J}=8.42 \mathrm{~Hz}, 1 \mathrm{H}$, pyridobenzimidazole $\left.\mathrm{C}_{9}-\mathrm{H}\right) \cdot{ }^{13} \mathrm{C}-\mathrm{NMR}\left(125 \mathrm{MHz}, \mathrm{DMSO}-\mathrm{d}_{6}, \delta \mathrm{ppm}\right)$ : $21.11\left(\mathrm{CH}_{3}\right), 42.95\left(\mathrm{NCH}_{2}\right), 44.84\left(\mathrm{CH}_{2} \mathrm{Cl}\right), 85.28$ (pyridobenzimidazole $\mathrm{C}_{4}$ ), 91.84 (pyridobenzimidazole $\mathrm{C}_{9}$ ), 115.18 (pyridobenzimidazole $\mathrm{C}_{2}$ ), $119.06(\mathrm{CN}), 118.62,120.47,126.11$ (pyridobenzimidazole $\mathrm{C}_{6,7.8}$ ), 128.25 (pyridobenzimidazole $\mathrm{C}_{9 \mathrm{a}}$ ), 145.36 (pyridobenzimidazole $\mathrm{C}_{4 \mathrm{a}}$ ), 148.52 (pyridobenzimidazole $\mathrm{C}_{5 \mathrm{a}}$ ), 149.19 (pyridobenzimidazole C3), 151.60 (pyridobenzimidazole C1). Anal. Calcd. for $\mathrm{C}_{15} \mathrm{H}_{13} \mathrm{ClN}_{4}$ (284.75): C, 63.27; H, 4.60; N, 19.68. Found: C, 63.49; H, 4.66; N, 19.85.

3-Methyl-1 - [2-(substituted)ethyl] aminopyrido[1,2-a] benzimidazole-4-carbonitrile (13a-e): A mixture of 1-(2-chloroethyl) amino-3-methylpyridobenzimidazole-4-carbonitrile (12) (2 mmol, $0.57 \mathrm{~g}$ ) and dimethylamine, tert-butylamine, piperidine, morpholine or piperazine $(6 \mathrm{mmol})$ respectively, in 10:8 mixture of dioxane/ dimethylformamide $(18 \mathrm{ml})$ was stirred at room temperature for 24 hrs in case of compounds $(\mathbf{1 3 a}, \mathbf{b})$ or at $60-80^{\circ} \mathrm{C}$ for $12 \mathrm{hrs}$ in case of compounds $(\mathbf{1 3 c} \mathbf{c}-\mathbf{e})$. Crushed ice was added to the reaction mixture and the separated product was filtered, washed with water and crystallized from the proper solvent.

1-[2-(Dimethylamino)ethyl]amino-3-methylpyrido[1,2-a] benzimidazole-4-carbonitrile (13a): Yield $37.29 \%$, M.P. $>258^{\circ} \mathrm{C}$; crystallization solvent: DMF/ethanol. IR $\left(\mathrm{KBr}, \mathrm{cm}^{-1}\right): 3382(\mathrm{NH}), 3036$, 3010, 2978, 2950, 2918, 2861, $2816(\mathrm{C}-\mathrm{H}), 2205(\mathrm{C} \equiv \mathrm{N}), 1632(\mathrm{C}=\mathrm{N})$, 1598, 1559, 1462, $1431(\mathrm{C}=\mathrm{C}) .{ }^{1} \mathrm{H}-\mathrm{NMR}\left(400 \mathrm{MHz}, \mathrm{DMSO}-\mathrm{d}_{6}\right) \delta(\mathrm{ppm})$ : $2.30\left(\mathrm{~s}, 6 \mathrm{H}, 2 \mathrm{CH}_{3}\right), 2.53\left(\mathrm{~s}, 3 \mathrm{H}, \mathrm{CH}_{3}\right.$ at $\left.\mathrm{C}_{3}\right), 2.69(\mathrm{t}, \mathrm{J}=6.29 \mathrm{~Hz}, 2 \mathrm{H}$, $\left.\mathrm{N}-\mathrm{CH}_{2}\right), 3.52\left(\mathrm{t}, \mathrm{J}=6.25 \mathrm{~Hz}, \mathrm{CH}_{2}-\mathrm{NH}\right), 6.15(\mathrm{~s}, 1 \mathrm{H}$, pyridobenzimidazole $\left.\mathrm{C}_{2}-\mathrm{H}\right), 7.28\left(\mathrm{~s}, 1 \mathrm{H}, \mathrm{NH}, \mathrm{D}_{2} \mathrm{O}\right.$ exchangeable), $7.34(\mathrm{t}, \mathrm{J}=7.80 \mathrm{~Hz}, 1 \mathrm{H}$, pyridobenzimidazole $\left.\mathrm{C}_{7}-\mathrm{H}\right), 7.53(\mathrm{t}, \mathrm{J}=7.80 \mathrm{~Hz}, 1 \mathrm{H}$, pyridobenzimidazole $\left.\mathrm{C}_{8}-\mathrm{H}\right), 7.79\left(\mathrm{~d}, \mathrm{~J}=8.32 \mathrm{~Hz}, 1 \mathrm{H}\right.$, pyridobenzimidazole $\left.\mathrm{C}_{6}-\mathrm{H}\right), 8.23$ (d, $\mathrm{J}=8.37 \mathrm{~Hz}, 1 \mathrm{H}$, pyridobenzimidazole $\mathrm{C}_{9}-\mathrm{H}$ ). Anal. Calcd. for $\mathrm{C}_{17} \mathrm{H}_{19} \mathrm{~N}_{5}(293.37)$ : C, 69.60; H, 6.53; N, 23.87. Found: C, 69.87; H, 6.59; $\mathrm{N}, 24.12$.

1-[2-(tert-Butylamino)ethyl] amino-3-methylpyrido[1,2-a] benzimidazole-4-carbonitrile (13b): Yield $78.13 \%$, M.P. $>286^{\circ} \mathrm{C}$; crystallization solvent: ethanol. IR $\left(\mathrm{KBr}, \mathrm{cm}^{-1}\right): 3536(\mathrm{NH}), 3302,3113$, $2966(\mathrm{C}-\mathrm{H}), 2210(\mathrm{C} \equiv \mathrm{N}), 1631(\mathrm{C}=\mathrm{N}), 1595(\mathrm{C}=\mathrm{C}) .{ }^{1} \mathrm{H}-\mathrm{NMR}(400$ MHz,DMSO-d $\mathrm{d}_{6} \delta$ (ppm): $1.21\left(\mathrm{~s}, 9 \mathrm{H}, 3 \mathrm{CH}_{3}\right), 2.54\left(\mathrm{~s}, 3 \mathrm{H}, \mathrm{CH}_{3}\right.$ at $\left.\mathrm{C}_{3}\right)$, 2.90-3.11 (m, $\left.2 \mathrm{H}, \mathrm{N}-\mathrm{CH}_{2}\right), 3.49-3.73\left(\mathrm{~m}, 2 \mathrm{H}, \mathrm{CH}_{2}-\mathrm{NH}\right), 6.25(\mathrm{~s}, 1 \mathrm{H}$, pyridobenzimidazole $\left.\mathrm{C}_{2}-\mathrm{H}\right), 7.32(\mathrm{t}, \mathrm{J}=7.47 \mathrm{~Hz}, 1 \mathrm{H}$, pyridobenzimidazole $\left.\mathrm{C}_{7}-\mathrm{H}\right), 7.53\left(\mathrm{t}, \mathrm{J}=7.47 \mathrm{~Hz}, 1 \mathrm{H}\right.$, pyridobenzimidazole $\left.\mathrm{C}_{8}-\mathrm{H}\right), 7.79(\mathrm{~d}$, $\mathrm{J}=8.05 \mathrm{~Hz}, 1 \mathrm{H}$, pyridobenzimidazole $\left.\mathrm{C}_{6}-\mathrm{H}\right), 8.45(\mathrm{~d}, \mathrm{~J}=8.18 \mathrm{~Hz}, 1 \mathrm{H}$, pyridobenzimidazole $\mathrm{C}_{9}-\mathrm{H}$ ). Anal. Calcd. for $\mathrm{C}_{19} \mathrm{H}_{23} \mathrm{~N}_{5}$ (321.43): C, 71.00; H, 7.21; N, 21.79. Found: C, 71.24; H, 7.28; N, 21.88 .

3-Methyl-1-[2-(piperidin-1-yl)ethyl] aminopyrido[1,2-a] benzimidazole-4-carbonitrile (13c): Yield $89.55 \%$, M.P. $>264^{\circ} \mathrm{C}$; crystallization solvent: dioxane/ ethanol. IR $\left(\mathrm{KBr}, \mathrm{cm}^{-1}\right)$ : $3360(\mathrm{NH})$, 3041, 3012, 2975, 2931, 2853, $2829(\mathrm{C}-\mathrm{H}), 2205(\mathrm{C} \equiv \mathrm{N}), 1633(\mathrm{C}=\mathrm{N})$, 1598, 1549, 1463 (C=C). ${ }^{1} \mathrm{H}-\mathrm{NMR}$ (400 MHz,DMSO-d $) \delta(\mathrm{ppm}): 1.39$ $1.51\left(\mathrm{~m}, 2 \mathrm{H}\right.$, piperidine $\left.\mathrm{C}_{4}-\mathrm{H}_{2}\right), 1.51-1.64\left(\mathrm{~m}, 4 \mathrm{H}\right.$, piperidine $\left.\mathrm{C}_{3,5}-\mathrm{H}_{2}\right)$, 2.50 (under DMSO, 7H, $\mathrm{CH}_{3}$ and piperidine $\left.\mathrm{C}_{26}-\mathrm{H}_{2}\right), 2.72(\mathrm{t}, \mathrm{J}=6.31$ $\mathrm{Hz}, 2 \mathrm{H}$, ethyl $\left.\mathrm{N}-\mathrm{CH}_{2}\right), 3.51\left(\mathrm{t}, \mathrm{J}=6.07 \mathrm{~Hz}, 2 \mathrm{H}, \mathrm{NCH}_{2}\right), 6.10(\mathrm{~s}, 1 \mathrm{H}$, pyridobenzimidazole $\left.\mathrm{C}_{2}-\mathrm{H}\right), 7.28-7.39(\mathrm{~m}, 2 \mathrm{H}$, pyridobenzimidazole $\mathrm{C}_{7}-\mathrm{H}$ and $\mathrm{NH}, \mathrm{D}_{2} \mathrm{O}$ exchangeable), $7.53(\mathrm{t}, 1 \mathrm{H}, \mathrm{J}=7.69 \mathrm{~Hz}$, pyridobenzimidazole $\left.\mathrm{C}_{8}-\mathrm{H}\right), 7.8(\mathrm{~d}, \mathrm{~J}=8.00 \mathrm{~Hz}, 1 \mathrm{H}$, pyridobenzimidazole
$\left.\mathrm{C}_{6}-\mathrm{H}\right), 8.26$ (d, J=8.25 Hz, 1H,pyridobenzimidazole $\mathrm{C}_{9}$-H).EI-Mass spectrum $\mathrm{m} / \mathrm{z}$ (relative abundance\%) 334.25 (0.91); 333.25 (3.25); 235.14 (1.64); 206.10 (1.03); 194.09 (0.87); 102.06 (0.79); 99.16 (6.71); 98.11 (100.00); 96.10 (1.72); 83.12 (0.85); 70.09(3.82); 55.07(2.03). Anal. Calcd. for $\mathrm{C}_{20} \mathrm{H}_{23} \mathrm{~N}_{5}$ (333.44): C, 72.04; H, 6.95; N, 21.00. Found: C, $72.18 ; \mathrm{H}, 7.02 ; \mathrm{N}, 21.17$.

3-Methyl-1-[2-(morpholin-4-yl)ethyl] aminopyrido[1,2-a] benzimidazole-4-carbonitrile (13d):

Yield $47.76 \%$, M.P. $>258^{\circ} \mathrm{C}$; crystallization solvent: dioxane. IR $\left(\mathrm{KBr}, \mathrm{cm}^{-1}\right): 3377(\mathrm{NH})$, 3070, 2957, 2834, $2803(\mathrm{C}-\mathrm{H}), 2201(\mathrm{C} \equiv \mathrm{N})$, $1629(\mathrm{C}=\mathrm{N}), 1596,1558,1458(\mathrm{C}=\mathrm{C}), 1265,1028(\mathrm{C}-\mathrm{O}-\mathrm{C}) .{ }^{1} \mathrm{H}-\mathrm{NMR}$ $\left(400 \mathrm{MHz}, \mathrm{DMSO}-\mathrm{d}_{6}\right) \delta(\mathrm{ppm}): 2.51\left(\mathrm{~s}, 3 \mathrm{H}, \mathrm{CH}_{3}\right), 2.55(\mathrm{t}, \mathrm{J}=4.44 \mathrm{~Hz}$, $4 \mathrm{H}$, morpholine $\left.\mathrm{C}_{3,5}-\mathrm{H}_{2}\right), 2.76\left(\mathrm{t}, \mathrm{J}=6.30 \mathrm{~Hz}, 2 \mathrm{H}, \mathrm{N}-\mathrm{CH}_{2}\right), 3.54(\mathrm{q}$, $\left.\mathrm{J}=6.2 \mathrm{~Hz}, 2 \mathrm{H}, \mathrm{CH}_{2}-\mathrm{NH}\right), 3.64\left(\mathrm{t}, \mathrm{J}=4.49 \mathrm{~Hz}, 4 \mathrm{H}\right.$, morpholine $\left.\mathrm{C}_{2,6}-\mathrm{H}_{2}\right)$, $6.13\left(\mathrm{~s}, 1 \mathrm{H}\right.$, pyridobenzimidazole $\left.\mathrm{C}_{2}-\mathrm{H}\right), 7.27(\mathrm{t}, \mathrm{J}=4.06 \mathrm{~Hz}, 1 \mathrm{H}, \mathrm{NH}$ , $\mathrm{D}_{2} \mathrm{O}$ exchangeable), $7.36(\mathrm{t}, \mathrm{J}=7.66 \mathrm{~Hz}, 1 \mathrm{H}$, pyridobenzimidazole $\left.\mathrm{C}_{7}-\mathrm{H}\right), 7.53\left(\mathrm{t}, \mathrm{J}=7.66 \mathrm{~Hz}, 1 \mathrm{H}\right.$, pyridobenzimidazole $\left.\mathrm{C}_{8}-\mathrm{H}\right), 7.8(\mathrm{~d}$, $\mathrm{J}=8.02 \mathrm{~Hz}, 1 \mathrm{H}$, pyridobenzimidazole $\left.\mathrm{C}_{6}-\mathrm{H}\right), 8.28(\mathrm{~d}, \mathrm{~J}=8.34 \mathrm{~Hz}, 1 \mathrm{H}$, pyridobenzimidazole $\mathrm{C}_{9}-\mathrm{H}$ ). Anal. Calcd. for $\mathrm{C}_{19} \mathrm{H}_{21} \mathrm{~N}_{5} \mathrm{O}$ (335.41): C, 68.04; H, 6.31; N, 20.88. Found: C, 68.31; H, 6.34; N, 21.06.

3-Methyl-1-[2-(piperazin-1-yl)ethyl] aminopyrido[1,2-a] benzimidazole-4-carbonitrile (13e):

Yield $74.63 \%$, M.P. $>276^{\circ} \mathrm{C}$; crystallization solvent: DMF/ $\mathrm{H}_{2} \mathrm{O}$. IR $\left(\mathrm{KBr}, \mathrm{cm}^{-1}\right): 3387,3206(\mathrm{NH}), 3073,3049,2945,2890,2814(\mathrm{C}-$ $\mathrm{H}), 2200(\mathrm{C} \equiv \mathrm{N}), 1630(\mathrm{C}=\mathrm{N}), 1596,1561,1458(\mathrm{C}=\mathrm{C}) .{ }^{1} \mathrm{H}-\mathrm{NMR}$ $\left(400 \mathrm{MHz}, \mathrm{DMSO}-\mathrm{d}_{6}\right) \delta(\mathrm{ppm}): 2.52\left(\mathrm{~s}, 3 \mathrm{H}, \mathrm{CH}_{3}\right), 2.57-2.68(\mathrm{~m}, 4 \mathrm{H}$, piperazine $\left.\mathrm{C}_{2,6}-\mathrm{H}_{2}\right), 2.77\left(\mathrm{t}, \mathrm{J}=6.22 \mathrm{~Hz}, 2 \mathrm{H}, \mathrm{N}-\mathrm{CH}_{2}\right), 2.93(\mathrm{t}, \mathrm{J}=4.66 \mathrm{~Hz}$, $4 \mathrm{H}$, piperazine $\left.\mathrm{C}_{3,5}-\mathrm{H}_{2}\right), 3.53\left(\mathrm{t}, \mathrm{J}=6.23 \mathrm{~Hz}, 2 \mathrm{H}, \mathrm{CH}_{2}-\mathrm{NH}\right), 6.13(\mathrm{~s}, 1 \mathrm{H}$, pyridobenzimidazole $\left.\mathrm{C}_{2}-\mathrm{H}\right), 7.34(\mathrm{t}, J=7.72 \mathrm{~Hz}, 1 \mathrm{H}$, pyridobenzimidazole $\left.\mathrm{C}_{7}-\mathrm{H}\right), 7.35\left(\mathrm{t}, J=7.72 \mathrm{~Hz}, 1 \mathrm{H}\right.$, pyridobenzimidazole $\left.\mathrm{C}_{8}-\mathrm{H}\right), 7.8(\mathrm{~d}$, $\mathrm{J}=8.05 \mathrm{~Hz}, 1 \mathrm{H}$, pyridobenzimidazole $\left.\mathrm{C}_{6}-\mathrm{H}\right), 8.3(\mathrm{~d}, \mathrm{~J}=8.29 \mathrm{~Hz}, 1 \mathrm{H}$, pyridobenzimidazole $\mathrm{C}_{9}-\mathrm{H}$ ). Anal. Calcd. for $\mathrm{C}_{19} \mathrm{H}_{22} \mathrm{~N}_{6}$ (334.43): C, 68.24; H, 6.63; N, 25.13. Found: C, 68.42; H, 6.68; N, 25.37

3-Methyl-1-(4-substituted

phenylamino)pyrido $[1,2-\mathrm{a}]$ benzimidazole-4-carbonitrile (14a-c): A mixture of the chloro derivative (10) (4 mmol, $0.97 \mathrm{~g}$ ) and the proper arylamines, (12 mmoles $)$ in dioxane $(20 \mathrm{ml})$ was heated at $60-80^{\circ} \mathrm{C}$ for $13-38 \mathrm{~h}$. The reaction mixture was then poured into ice cold water. The product was filtered, washed with water, dried and crystallized from DMF/ethanol.

1 - (4-Hydroxyphenyl) a mino-3 - methylpyrido [1,2-a] benzimidazole-4-carbonitrile (14a): Yield $71.58 \%$, M.P. $>300^{\circ} \mathrm{C}$; IR $\left(\mathrm{KBr}, \mathrm{cm}^{-1}\right): 3368(\mathrm{OH}), 3100(\mathrm{NH}), 3006,2929,2875(\mathrm{C}-$ $\mathrm{H}), 2209(\mathrm{C} \equiv \mathrm{N}), 1626(\mathrm{C}=\mathrm{N}), 1592,1544,1512,1461 \quad(\mathrm{C}=\mathrm{C})$. ${ }^{1} \mathrm{H}-\mathrm{NMR} \quad\left(400 \mathrm{MHz}, \mathrm{DMSO}-\mathrm{d}_{6}\right) \quad \delta \quad(\mathrm{ppm}): 2.42 \quad\left(\mathrm{~s}, 3 \mathrm{H}, \mathrm{CH}_{3}\right)$, $5.91(\mathrm{~s}, 1 \mathrm{H}$, pyridobenzimidazole $\mathrm{C} 2-\mathrm{H}), 6.88(\mathrm{~d}, \mathrm{~J}=7.98 \mathrm{~Hz}, 2 \mathrm{H}$ 4-hydroxyphenyl $\left.\mathrm{C}_{26}-\mathrm{H}\right), 7.23(\mathrm{~d}, \mathrm{~J}=7.98 \mathrm{~Hz}, 2 \mathrm{H}$, 4-hydroxyphenyl $\left.\mathrm{C}_{3,5}-\mathrm{H}\right), 7.32\left(\mathrm{t}, \mathrm{J}=7.70 \mathrm{~Hz}, 1 \mathrm{H}\right.$, pyridobenzimidazole $\left.\mathrm{C}_{7}-\mathrm{H}\right), 7.53$ $\left(\mathrm{t}, \mathrm{J}=7.70 \mathrm{~Hz}, 1 \mathrm{H}\right.$, pyridobenzimidazole $\left.\mathrm{C}_{8}-\mathrm{H}\right), 7.80(\mathrm{~d}, \mathrm{~J}=7.84$ $\mathrm{Hz}, 1 \mathrm{H}$, pyridobenzimidazole $\left.\mathrm{C}_{6}-\mathrm{H}\right), 8.49(\mathrm{~d}, \mathrm{~J}=8.00 \mathrm{~Hz}, 1 \mathrm{H}$, pyridobenzimidazole $\left.\mathrm{C}_{9}-\mathrm{H}\right), 9.09,9.57\left(2 \mathrm{~s}\right.$, each $1 \mathrm{H}, \mathrm{NH}$ and $\mathrm{OH}, \mathrm{D}_{2} \mathrm{O}$ exchangeable). Anal. Calcd. for $\mathrm{C}_{19} \mathrm{H}_{14} \mathrm{~N}_{4} \mathrm{O}$ (314.35): C, 72.60; H, 4.49; $\mathrm{N}, 17.82$. Found: C, 72.78; H, 4.58; N, 18.01 .

1 - (4-Methoxyphenyl)amino-3-methylpyrido [1,2-a] benzimidazole-4-carbonitrile (14b): Yield $95.20 \%$, M.P. $>300^{\circ} \mathrm{C}$; IR $\left(\mathrm{KBr}, \mathrm{cm}^{-1}\right): 3367(\mathrm{NH}), 3073,3052,3010,2950,2909,2830(\mathrm{C}-$ $\mathrm{H}), 2205(\mathrm{C} \equiv \mathrm{N}), 1629(\mathrm{C}=\mathrm{N}), 1593,1546,1512,1473(\mathrm{C}=\mathrm{C}), 1263$, 1029 (C-O-C). ${ }^{1} \mathrm{H}-\mathrm{NMR}$ (400 MHz,DMSO-d $)_{6} \delta(\mathrm{ppm}): 2.42(\mathrm{~s}$, $\left.3 \mathrm{H}, \mathrm{CH}_{3}\right), 3.80\left(\mathrm{~s}, 3 \mathrm{H}, \mathrm{O}-\mathrm{CH}_{3}\right), 5.98(\mathrm{~s}, 1 \mathrm{H}$, pyridobenzimidazole 
Citation: Darwish SAZ, Elbayaa RY, Ashour HMA, Khalil MA, Badawey EAM (2018) Potential Anticancer Agents: Design, Synthesis of New Pyrido[1,2-a]benzimidazoles and Related Derivatives Linked to Alkylating Fragments. Med Chem (Los Angeles) 8: 86-095. doi: 10.4172/21610444.1000498

$\left.\mathrm{C}_{2}-\mathrm{H}\right), 7.04\left(\mathrm{~d}, \mathrm{~J}=8.5 \mathrm{~Hz}, 2 \mathrm{H}\right.$, methoxyphenyl $\left.\mathrm{C}_{2,6}-\mathrm{H}\right), 7.29-7.36(\mathrm{~m}$, $3 \mathrm{H}$, pyridobenzimidazole $\mathrm{C}_{7}-\mathrm{H}$ and methoxyphenyl $\left.\mathrm{C}_{3,5}-\mathrm{H}\right), 7.54$ $\left(\mathrm{t}, \mathrm{J}=7.64 \mathrm{~Hz}, 1 \mathrm{H}\right.$ of pyridobenzimidazole $\left.\mathrm{C}_{8}-\mathrm{H}\right), 7.81(\mathrm{~d}, \mathrm{~J}=6.75$ $\mathrm{Hz}, 1 \mathrm{H}$, pyridobenzimidazole $\left.\mathrm{C}_{6}-\mathrm{H}\right), 8.48(\mathrm{~d}, \mathrm{~J}=7.435 \mathrm{~Hz}, 1 \mathrm{H}$, pyridobenzimidazole $\left.\mathrm{C}_{9}-\mathrm{H}\right), 9.2\left(\mathrm{~s}, 1 \mathrm{H}, \mathrm{NH}, \mathrm{D}_{2} \mathrm{O}\right.$ exchangeable). ${ }^{13} \mathrm{C}-\mathrm{NMR}$ (125 MHz, DMSO-d6, $\left.\delta \mathrm{ppm}\right): 21.02\left(\mathrm{CH}_{3}\right), 55.73\left(\mathrm{OCH}_{3}\right)$, 87.15 (pyridobenzimidazole $\mathrm{C}_{4}$ ), 95.32 (pyridobenzimidazole $\mathrm{C}_{9}$ ), 115.36 (methoxyphenyl $\mathrm{C}_{2,6}$ ), 116.24 (pyridobenzimidazole $\mathrm{C}_{2}$ ), $116.97(\mathrm{CN}), 118.77,120.56,126.11$ (pyridobenzimidazole $\mathrm{C}_{6.8}$ ), 126.27 (methoxyphenyl $\mathrm{C}_{3,5}$ ), 128.80 (pyridobenzimidazole $\mathrm{C}_{9 \mathrm{a}}$ ), 131.96 (methoxyphenyl $\mathrm{C}_{1}$ ), 145.15 (pyridobenzimidazole $\mathrm{C}_{4 \mathrm{a}}$ ), 148.92 (pyridobenzimidazole $\mathrm{C}_{5 \mathrm{a}}$ ), 149.16 (methoxyphenyl $\mathrm{C}_{4}$ ), 151.15 (pyridobenzimidazole $\mathrm{C}_{3}$ ), 157.62 (pyridobenzimidazole $\mathrm{C}_{1}$ ). Anal. Calcd. for $\mathrm{C}_{20} \mathrm{H}_{16} \mathrm{~N}_{4} \mathrm{O}$ (328.38): C, 73.15; H, 4.91; N, 17.06. Found: C, 73.24; $\mathrm{H}, 4.98 ; \mathrm{N}, 17.14$.

3-Methyl-1-[3-(trifluoromethyl)phenylamino]pyrido[1,2-a] benzimidazole-4-carbonitrile (14c): Yield $68.21 \%$, M.P. $299^{\circ} \mathrm{C}$; IR $\left(\mathrm{KBr}, \mathrm{cm}^{-1}\right): 3469(\mathrm{NH}), 2211(\mathrm{C} \equiv \mathrm{N}), 1636(\mathrm{C}=\mathrm{N}), 1548,1454(\mathrm{C}=\mathrm{C})$. ${ }^{1} \mathrm{H}-\mathrm{NMR}\left(400 \mathrm{MHz}, \mathrm{DMSO}-\mathrm{d}_{6}\right) \delta(\mathrm{ppm}): 2.4\left(\mathrm{~s}, 3 \mathrm{H}, \mathrm{CH}_{3}\right), 6.31(\mathrm{~s}, 1 \mathrm{H}$, pyridobenzimidazole $\left.\mathrm{C}_{2}-\mathrm{H}\right), 7.28-7.85(\mathrm{~m}, 7 \mathrm{H}$, pyrido-benzimidazole $\mathrm{C}_{6,7,8}-\mathrm{H}$ and trifluoromethylphenyl $\left.\mathrm{C}_{2,4,5,6}-\mathrm{H}\right), 8.51(\mathrm{~d}, \mathrm{~J}=8.00 \mathrm{~Hz}, 1 \mathrm{H}$, pyrido-benzimidazole $\left.\mathrm{C}_{9}-\mathrm{H}\right), 9.69\left(\mathrm{~s}, 1 \mathrm{H}, \mathrm{NH}, \mathrm{D}_{2} \mathrm{O}\right.$ exchangeable). Anal. Calcd. for $\mathrm{C}_{20} \mathrm{H}_{13} \mathrm{~F}_{3} \mathrm{~N}_{4}$ (366.35): C, 65.57; H, 3.58; N, 15.29. Found: C, $65.72 ; \mathrm{H}, 3.56 ; \mathrm{N}, 15.43$.

1-Dimethylamino-3-methylpyrido[1,2-a]benzimidazole-4carbonitrile (15): A mixture of the chloro derivative (10) (4 mmol, 0.97 g) and dimethylamine, $(12$ mmoles, $\mathrm{g})$ in dioxane $(20 \mathrm{ml})$ was stirred at room temperature for $31 \mathrm{~h}$. The reaction mixture was then poured into ice cold water. The product was filtered, washed with water, dried and crystallized from dioxane. Yield $94.88 \%$, M.P. $>257^{\circ} \mathrm{C}$; IR $\left(\mathrm{KBr}, \mathrm{cm}^{-1}\right)$ : 3044, 3009, 2952, 2882, $2850(\mathrm{C}-\mathrm{H}), 2218(\mathrm{C} \equiv \mathrm{N}), 1626(\mathrm{C}=\mathrm{N}), 1593$, 1511, 1481, 1442 (C=C). ${ }^{1} \mathrm{H}-\mathrm{NMR}\left(400 \mathrm{MHz}, \mathrm{DMSO}_{-} \mathrm{d}_{6}\right) \delta(\mathrm{ppm}): 2.60$ $\left(\mathrm{s}, 3 \mathrm{H}, \mathrm{CH}_{3}\right.$ at $\left.\mathrm{C}_{3}\right), 2.93\left(\mathrm{~s}, 6 \mathrm{H}, 2 \mathrm{CH}_{3}\right), 6.59(\mathrm{~s}, 1 \mathrm{H}$, pyridobenzimidazole $\left.\mathrm{C}_{2}-\mathrm{H}\right), 7.37\left(\mathrm{t}, \mathrm{J}=7.72 \mathrm{~Hz}, 1 \mathrm{H}\right.$, pyridobenzimidazole $\left.\mathrm{C}_{7}-\mathrm{H}\right), 7.54$ $\left(\mathrm{t}, \mathrm{J}=7.72 \mathrm{~Hz}, 1 \mathrm{H}\right.$, pyridobenzimidazole $\left.\mathrm{C}_{8}-\mathrm{H}\right), 7.83(\mathrm{~d}, \mathrm{~J}=8.56$ $\mathrm{Hz}, 1 \mathrm{H}$, pyridobenzimidazole $\left.\mathrm{C}_{6}-\mathrm{H}\right), 8.14(\mathrm{~d}, \mathrm{~J}=8.33 \mathrm{~Hz}, 1 \mathrm{H}$, pyridobenzimidazole $\mathrm{C}_{9}-\mathrm{H}$ ). Anal. Calcd. for $\mathrm{C}_{15} \mathrm{H}_{14} \mathrm{~N}_{4}$ (250.31): C, 71.98; H, 5.64; N, 22.38. Found: C, 72.15; H, 5.73; N, 22.51.

1 - Bis (2-hydroxyethyl)amino-3-methylpyrido [1,2-a] benzimidazole-4-carbonitrile (16): A mixture of the chloro derivative (10) $(4 \mathrm{mmol}, 0.97 \mathrm{~g})$ and diethanolamine (12 mmoles) in dioxane (20 $\mathrm{ml}$ ) was heated at $60-80^{\circ} \mathrm{C}$ for 27 . The reaction mixture was then poured into ice cold water. The product was filtered, washed with water, dried and crystallized from DMF $/ \mathrm{H}_{2} \mathrm{O}$. Yield $82.26 \%$, M.P. $257^{\circ} \mathrm{C} ; \mathrm{IR}(\mathrm{KBr}, \mathrm{cm}$ $\left.{ }^{1}\right)$ : $3381(\mathrm{OH}), 2976,2927,2867(\mathrm{C}-\mathrm{H}), 2214(\mathrm{C} \equiv \mathrm{N}), 1624(\mathrm{C}=\mathrm{N}), 1591$, 1512, 1477, $1432(\mathrm{C}=\mathrm{C}) .{ }^{1} \mathrm{H}-\mathrm{NMR}\left(400 \mathrm{MHz}, \mathrm{DMSO}-\mathrm{d}_{6}\right) \delta(\mathrm{ppm}): 2.58$ $\left(\mathrm{s}, 3 \mathrm{H}, \mathrm{CH}_{3}\right), 3.36-3.69\left(\mathrm{~m}, 8 \mathrm{H}, 2 \mathrm{~N}-\mathrm{CH}_{2}\right.$ and $\left.2 \mathrm{CH}_{2}-\mathrm{O}\right), 4.56(\mathrm{t}, \mathrm{J}=4.40$ $\mathrm{Hz}, 2 \mathrm{H}, 2 \mathrm{OH}, \mathrm{D}_{2} \mathrm{O}$ exchangeable), $6.69(\mathrm{~s}, 1 \mathrm{H}$, pyridobenzimidazole $\left.\mathrm{C}_{2}-\mathrm{H}\right), 7.34\left(\mathrm{t}, \mathrm{J}=7.69 \mathrm{~Hz}, 1 \mathrm{H}\right.$, pyridobenzimidazole $\left.\mathrm{C}_{7}-\mathrm{H}\right), 7.52$ $\left(\mathrm{t}, \mathrm{J}=7.69 \mathrm{~Hz}, 1 \mathrm{H}\right.$, pyridobenzimidazole $\left.\mathrm{C}_{8}-\mathrm{H}\right), 7.81(\mathrm{~d}, \mathrm{~J}=8.06$ $\mathrm{Hz}, 1 \mathrm{H}$, pyridobenzimidazole $\left.\mathrm{C}_{6}-\mathrm{H}\right), 8.20(\mathrm{~d}, \mathrm{~J}=8.38 \mathrm{~Hz}, 1 \mathrm{H}$, pyridobenzimidazole $\mathrm{C}_{9}-\mathrm{H}$ ). Anal. Calcd. for $\mathrm{C}_{17} \mathrm{H}_{18} \mathrm{~N}_{4} \mathrm{O}_{2}$ (310.36): C, 65.79; H, 5.85; N, 18.05. Found: C, 66.01; H, 5.94; N, 18.31 .

1 - B is ( 2-chloroethyl)amino-3-methylpyrido[1,2-a] benzimidazole-4-carbonitrile (17): A suspension of 1-bis(2hydroxyethyl)amino-3-methylpyrido [1,2-a]benzimidazole-4carbonitrile (16) $(5 \mathrm{mmol})$ in phosphorous oxychloride $(5 \mathrm{ml})$ was heated under reflux while stirring for $3 \mathrm{~h}$. The reaction mixture was allowed to cool to room temperature, then poured onto crushed ice and neutralized with $\mathrm{Na}_{2} \mathrm{CO}_{3}$. The product was filtered, washed with water and crystallized from dioxane/ ethanol. Yield $89.93 \%$, M.P. $237^{\circ} \mathrm{C}$; IR $\left(\mathrm{KBr}, \mathrm{cm}^{-1}\right): 3055,3020,2961,2920,2874(\mathrm{C}-\mathrm{H}), 2214(\mathrm{C} \equiv \mathrm{N})$, $1624(\mathrm{C}=\mathrm{N}), 1593,1511,1442(\mathrm{C}=\mathrm{C}) .{ }^{1} \mathrm{H}-\mathrm{NMR}\left(400 \mathrm{MHz}, \mathrm{DMSO}-\mathrm{d}_{6}\right)$ $\delta(\mathrm{ppm}): 2.60\left(\mathrm{~s}, 3 \mathrm{H}, \mathrm{CH}_{3}\right), 3.63-3.96\left(\mathrm{~m}, 8 \mathrm{H}, 2 \mathrm{NCH}_{2}\right.$ and $2 \mathrm{CH}_{2}-$ $\mathrm{Cl}), 6.88\left(\mathrm{~s}, 1 \mathrm{H}\right.$, pyridobenzimidazole $\left.\mathrm{C}_{2}-\mathrm{H}\right), 7.38(\mathrm{t}, J=7.72 \mathrm{~Hz}, 1 \mathrm{H}$, pyridobenzimidazole $\left.\mathrm{C}_{7}-\mathrm{H}\right), 7.55(\mathrm{t}, J=7.72 \mathrm{~Hz}, 1 \mathrm{H}$, pyridobenzimidazole $\left.\mathrm{C}_{8}-\mathrm{H}\right), 7.85\left(\mathrm{~d}, \mathrm{~J}=8.20 \mathrm{~Hz}, 1 \mathrm{H}\right.$, pyridobenzimidazole $\left.\mathrm{C}_{6}-\mathrm{H}\right), 8.23$ (d, J=8.83 Hz, $1 \mathrm{H}$, pyridobenzimidazole $\mathrm{C}_{9}-\mathrm{H}$ ). Anal. Calcd. For $\mathrm{C}_{17} \mathrm{H}_{16} \mathrm{C}_{12} \mathrm{~N}_{4}$ (347.24): C, 58.80; H, 4.64; N, 16.14. Found: C, 58.97; H, $4.61 ; \mathrm{N}, 16.32$.

\section{Antitumor activity}

Nine compounds 5b, 13a, 13d ,13e,14b, 14c, 15, 16, and 17 were selected by the National Cancer Institute (NCI), Bethseda, Maryland, USA to be evaluated for their in vitro antitumor activity. Effective onedose assay has been added to the NCI 60 Cell screen in order to increase compound throughput and reduce data turnaround time to suppliers while maintaining efficient identification of active compounds. All the selected compounds were tested initially at a single dose $(10 \mu \mathrm{M})$ in the full NCI 60 cell panel including leukemia, non-small cell lung, colon, CNS, melanoma, ovarian, renal, prostate and breast cancer cell lines. The results are presented in Table 1 as growth $(G \%)$ and only compounds which satisfy pre-determined threshold inhibition criteria would progress to the five-dose screen [26-29].

\section{Results and Discussion}

\section{Chemistry}

The synthetic procedures implemented to obtain the newly synthesized compounds are demonstrated in Schemes 1 and 2.

In Scheme 1, the enamine tautomers of Schiff bases 2, 3, 6 and $\mathbf{8}$ were synthesized by reacting the aldehyde $\mathbf{1}$ [7] with the proper amine. ${ }^{1} \mathrm{HNMR}$ spectrum of compound $\mathbf{2}$ displayed a singlet at $1.50 \mathrm{ppm}$ due to tert-butyl protons and a doublet at $8.35 \mathrm{ppm}$ integrated for one proton attributed to the methine proton. It also revealed one $\mathrm{D}_{2} \mathrm{O}$ exchangeable doublet at $11.60 \mathrm{ppm}$ characteristic for $\mathrm{NH}$ proton. Meanwhile, the ${ }^{1} \mathrm{HNMR}$ spectrum of compound 3 revealed one triplet at $3.69 \mathrm{ppm}$ attributed to the four protons of the ethanolamine side chain and $\mathrm{D}_{2} \mathrm{O}$ exchangeable triplet at $5.07 \mathrm{ppm}$ due to $\mathrm{OH}$ proton. One doublet at $8.46 \mathrm{ppm}$ integrated for one proton representing the methine proton. Also its ${ }^{13} \mathrm{CNMR}$ spectrum showed peaks at around 53 and $60 \mathrm{ppm}$ corresponding to $\mathrm{NCH}_{2}$ and $\mathrm{OCH}_{2}$ moieties of the ethanolamine side chain. ${ }^{1} \mathrm{HNMR}$ spectrum of compound $\mathbf{6}$ revealed a singlet at $1.42 \mathrm{ppm}$ due to the six protons of the two $\mathrm{CH}_{3}$ groups of the side chain and a doublet at $3.50 \mathrm{ppm}$ corresponding to the $\mathrm{CH}_{2}-\mathrm{O}$ protons. In addition, a $\mathrm{D}_{2} \mathrm{O}$ exchangeable triplet at $5.53 \mathrm{ppm}$ due to $\mathrm{OH}$ proton was observed. The spectrum also showed one doublet at $8.31 \mathrm{ppm}$ attributed to the methine proton and a doublet at $11.68 \mathrm{ppm}$ characteristic for the $\mathrm{NH}$ proton. Additionally, ${ }^{1} \mathrm{HNMR}$ spectrum of compound 8 revealed a singlet at $1.36 \mathrm{ppm}$ due to the $\mathrm{CH}_{3}$ protons of the side chain and $\mathrm{D}_{2} \mathrm{O}$ exchangeable triplet at $5.41 \mathrm{ppm}$ due to two $\mathrm{OH}$ protons. Two doublets at 8.32 and $11.70 \mathrm{ppm}$, each integrated for one proton attributed to the methine and the $\mathrm{NH}$ protons, respectively were shown.

Chlorination of aliphatic $\mathrm{OH}$ group was achieved by heating the starting material 3, 6 and $\mathbf{8}$ in excess phosphorous oxychloride to give the corresponding chlorinated compounds 4, 7 and 9 . The ${ }^{1} \mathrm{HNMR}$ spectrum of compound 4 lacked the $\mathrm{D}_{2} \mathrm{O}$ exchangeable triplet of the $\mathrm{OH}$ proton and revealed a doublet at $8.52 \mathrm{ppm}$ attributed to the 
Citation: Darwish SAZ, Elbayaa RY, Ashour HMA, Khalil MA, Badawey EAM (2018) Potential Anticancer Agents: Design, Synthesis of New Pyrido[1,2-a]benzimidazoles and Related Derivatives Linked to Alkylating Fragments. Med Chem (Los Angeles) 8: 86-095. doi: 10.4172/21610444.1000498

\begin{tabular}{|c|c|c|c|c|c|c|c|c|c|c|c|c|c|c|c|c|c|c|c|c|c|c|}
\hline \multirow[t]{2}{*}{$\begin{array}{l}\text { Comp } \\
\text { No. }\end{array}$} & \multicolumn{2}{|c|}{ Leukemia } & \multicolumn{3}{|c|}{$\begin{array}{c}\text { Non-Small Cell Lung } \\
\text { Cancer }\end{array}$} & \multicolumn{2}{|c|}{ Colon Cancer } & \multicolumn{3}{|c|}{ CNS Cancer } & \multicolumn{3}{|c|}{ Melanoma } & \multirow{2}{*}{\begin{tabular}{|l|}
$\begin{array}{l}\text { Ovarian } \\
\text { Cancer }\end{array}$ \\
SK- OV-3
\end{tabular}} & \multicolumn{3}{|c|}{ Renal Cancer } & \multirow{2}{*}{\begin{tabular}{|c|} 
PC \\
PC- 3 \\
\end{tabular}} & \multicolumn{4}{|c|}{ Breast Cancer } \\
\hline & $\begin{array}{l}\text { HL- } 60 \\
\text { (TB) }\end{array}$ & $\begin{array}{c}\text { RPMI- } \\
8226\end{array}$ & $\begin{array}{l}\text { A549/ } \\
\text { ATCC }\end{array}$ & $\begin{array}{c}\text { HOP- } \\
62\end{array}$ & $\begin{array}{l}\mathrm{NCl}- \\
\mathrm{H} 522\end{array}$ & $\begin{array}{c}\text { HCT- } \\
116\end{array}$ & HT29 & $\begin{array}{l}\text { SNB- } \\
19\end{array}$ & $\begin{array}{l}\text { SNB- } \\
75\end{array}$ & U251 & $\begin{array}{l}\text { LOX } \\
\text { IMVI }\end{array}$ & $\begin{array}{c}\text { SK- } \\
\text { MEL- } 5\end{array}$ & $\begin{array}{l}\text { UACC } \\
-62\end{array}$ & & $\begin{array}{c}786- \\
0\end{array}$ & CAKI- 1 & $\begin{array}{l}\text { UO- } \\
31\end{array}$ & & MCF 7 & $\begin{array}{c}\text { MDA- } \\
\text { MB- } \\
231 / \\
\text { ACTCC }\end{array}$ & $\begin{array}{l}\text { BT- } \\
549\end{array}$ & T-47D \\
\hline $5 b$ & 90.61 & 96.51 & 97.75 & 90.76 & 93.55 & 95.44 & 98.19 & 97.25 & 83.72 & 95.67 & 92.41 & 97.03 & 92.28 & 98.36 & 106.13 & 9.03 & 79.55 & 88.18 & 86.44 & 93.83 & 91.19 & 86.26 \\
\hline $13 a$ & 91.62 & 92.00 & 96.43 & 87.18 & 97.86 & 97.20 & 101.65 & 90.08 & 94.77 & 96.58 & 89.05 & 100.64 & 81.60 & 97.87 & 98.30 & 89.91 & 80.97 & 93.28 & 98.55 & 84.44 & 90.84 & 88.55 \\
\hline 13d & 97.26 & 92.87 & 83.42 & 93.43 & 72.54 & 90.60 & 89.72 & 115.47 & 73.27 & 95.36 & 99.56 & 93.57 & 110.15 & 88.23 & 92.90 & 85.61 & 82.36 & 82.44 & 88.74 & 104.11 & 87.01 & 68.31 \\
\hline $13 e$ & 91.60 & 95.78 & 87.59 & 84.71 & 85.52 & 75.01 & 84.65 & 100.01 & 83.41 & 90.71 & 99.29 & 95.30 & 94.46 & 82.51 & 86.64 & 93.00 & 85.67 & 88.12 & 86.84 & 94.11 & 83.52 & 89.04 \\
\hline $14 b$ & 43.63 & 93.34 & 72.60 & 79.96 & 82.00 & 77.64 & 98.55 & 98.11 & 95.65 & 85.60 & 94.41 & 103.67 & 86.58 & 90.41 & 105.31 & 100.31 & 88.24 & 77.45 & 90.16 & 93.42 & 87.45 & 108.08 \\
\hline $14 c$ & 31.80 & 22.82 & 43.73 & -2.32 & 20.69 & 39.61 & 13.19 & 40.70 & 59.41 & 11.62 & 59.64 & 4.24 & 11.90 & 46.41 & 58.95 & 38.74 & 28.32 & 21.80 & 36.31 & 54.18 & 19.39 & 8.68 \\
\hline 15 & 101.30 & 103.96 & 97.24 & 96.90 & 97.98 & 101.40 & 109.59 & 95.59 & 90.68 & 98.10 & 102.37 & 105.95 & 90.63 & 97.48 & 94.27 & 99.21 & 93.44 & 102.40 & 98.18 & 93.53 & 99.25 & 90.71 \\
\hline 16 & 85.53 & 85.36 & 94.77 & 90.03 & 83.88 & 22.15 & 86.43 & 92.78 & 79.85 & 98.81 & 84.85 & 92.56 & 76.98 & 101.95 & 83.66 & 90.37 & 81.23 & 88.87 & 77.13 & 81.11 & 97.73 & 82.20 \\
\hline 17 & 113.20 & 95.29 & 66.70 & 80.34 & 69.49 & 75.33 & 83.41 & 111.75 & 82.92 & 90.68 & 101.06 & 95.37 & 95.25 & 88.29 & 80.56 & 82.60 & 77.15 & 81.89 & 92.84 & 107.73 & 77.67 & 65.75 \\
\hline
\end{tabular}

"<20\%, Significant; $20-<40 \%$, Remarkable; $40-<60 \%$, Considerable; $60-<90 \%$, Weak

Table 1: The growth percentage (G \%) in single-dose assay for the selected compounds ${ }^{*}$

methine proton. The spectrum also showed a multiplet at around 11.2 ppm characteristic for the $\mathrm{NH}$ proton. Furthermore, the ${ }^{13} \mathrm{CNMR}$ spectrum displayed peaks at 44.15 and $52.02 \mathrm{ppm}$ attributed to $\mathrm{NCH}_{2}$ and $\mathrm{CH}_{2} \mathrm{Cl}$ moieties of the side chain, respectively. Nucleophilic displacement of aliphatic chlorine atom by aliphatic amines was achieved by reacting a suspension of $\mathbf{4}$ and the proper amine in a mixture of absolute ethanol and dimethylformamide to furnish compounds 5a- c. their IR spectrum showed absorption bands between $3227-3420 \mathrm{~cm}^{-1}$ corresponding to the NH groups. ${ }^{1} \mathrm{HNMR}$ spectrum of compound 5 a revealed a singlet at $1.50 \mathrm{ppm}$ representing the nine protons of the tert-butyl group and one doublet at $8.36 \mathrm{ppm}$ corresponding to the methine proton. A mulitplet at $11.61 \mathrm{ppm}$ attributed to the enamine $\mathrm{NH}$ proton was also displayed whereas, ${ }^{1} \mathrm{HNMR}$ spectrum of compound 5b revealed two multiplets at 1.40-1.53 and 2.44$2.50 \mathrm{ppm}$ corresponding to the piperidine protons. The spectrum also showed one doublet at $8.46 \mathrm{ppm}$ integrated for one proton attributed to the methine proton. A mulitplet at $11.18 \mathrm{ppm}$ attributed to the enamine $\mathrm{NH}$ proton was also displayed. Furthermore, the ${ }^{1} \mathrm{HNMR}$ spectrum of compound $\mathbf{5 c}$ showed two multiplets due to the morpholine protons, one doublet at $8.47 \mathrm{ppm}$ attributed to the methine proton and a mulitplet at $11.20 \mathrm{ppm}$ attributed to the enamine $\mathrm{NH}$ proton. ${ }^{1} \mathrm{HNMR}$ spectrum of the chlorinated compound 7 also lacked the $\mathrm{D}_{2} \mathrm{O}$ exchangeable triplet of the $\mathrm{OH}$ proton and revealed one doublet at $8.32 \mathrm{ppm}$ characteristic for the methine proton. $\mathrm{A}_{2} \mathrm{O}$ exchangeable doublet at $11.60 \mathrm{ppm}$ attributed to the $\mathrm{NH}$ was also displayed. Moreover, ${ }^{1} \mathrm{HNMR}$ spectrum of compound 9 lacked the $\mathrm{D}_{2} \mathrm{O}$ exchangeable triplet of the two $\mathrm{OH}$ protons and revealed one doublet at $8.35 \mathrm{ppm}$ integrated for one proton characteristic for the methine proton. A doublet at $11.63 \mathrm{ppm}$ attributed to the $\mathrm{NH}$ proton was also displayed.

In Scheme 2, the following amines 11, 14a-c and 16 were prepared by reacting the chloro derivative 10 [7] with ethanolamine, substituted aryl amines and diethanolamine in dioxane at $60-80^{\circ} \mathrm{C}$. ${ }^{1}$ HNMR spectrum of compound 11 revealed two quartets at 3.55 and $3.76 \mathrm{ppm}$ representing the four protons of ethanolamine moiety. The presence of both the $\mathrm{OH}$ proton and the $\mathrm{NH}$ proton was confirmed by the presence of two $\mathrm{D}_{2} \mathrm{O}$ exchangeable triplets at 5.01 and $7.24 \mathrm{ppm}$, respectively. While ${ }^{1} \mathrm{HNMR}$ spectrum of compound $\mathbf{1 4 b}$ showed a singlet at $3.80 \mathrm{ppm}$ representing the $\mathrm{OCH}_{3}$ protons and a doublet and a multiplet at 7.04 and around $7.3 \mathrm{ppm}$, respectively, indicating the presence of the 4-methoxyphenyl group. Also the ${ }^{13} \mathrm{CNMR}$ spectrum displayed signals at around 55.73, 115.36 and $126.27 \mathrm{ppm}$ attributed to $\mathrm{OCH}_{3}$ carbon and methoxyphenyl $\mathrm{C}_{2,6}$ and $\mathrm{C}_{3,5}$ respectively. In addition, peaks at 131.96 and $149.16 \mathrm{ppm}$ corresponding to methoxyphenyl $\mathrm{C}_{1}$ and $\mathrm{C}_{4}$ were observed. ${ }^{1} \mathrm{HNMR}$ spectrum of compound 16 revealed multiplet at 3.36-3.69 ppm representing the eight protons of the diethanolamine group. The presence of both $\mathrm{OH}$ protons was confirmed by the presence of a $\mathrm{D}_{2} \mathrm{O}$ exchangeable triplet at $4.56 \mathrm{ppm}$. The hydroxyl precursors $\mathbf{1 1}$ and $\mathbf{1 6}$ were chlorinated by heating in excess phosphorous oxychloride to produce compounds 12 and 17. Their IR spectra lacked the absorption bands attributed to the $\mathrm{OH}$ groups. In addition; their ${ }^{1} \mathrm{HNMR}$ spectra revealed disappearance of the $\mathrm{D}_{2} \mathrm{O}$ exchangeable $\mathrm{OH}$ proton signal confirming its replacement by the chloro group. ${ }^{1} \mathrm{HNMR}$ spectrum of compound $\mathbf{1 2}$ revealed a quartet at $3.85 \mathrm{ppm}$ and a triplet at $3.98 \mathrm{ppm}$ representing the four protons of the chloroethyl group. Its ${ }^{13} \mathrm{CNMR}$ spectrum showed peaks at 42.95 and $44.84 \mathrm{ppm}$ corresponding to $\mathrm{NCH}_{2}$ and $\mathrm{CH}_{2} \mathrm{Cl}$, respectively. Scheme 2 also describes the reaction of chloro derivative 12 with dimethylamine, tert-butylamine, piperidine, morpholine and piperazine to give the corresponding amines 13 a-e, respectively. Their IR spectra showed absorption bands in the range between 3206 and $3536 \mathrm{~cm}^{-1}$ corresponding to the NH groups. ${ }^{1} \mathrm{HNMR}$ spectrum of compound 13a revealed a singlet at $2.30 \mathrm{ppm}$ integrated for six protons indicating the presence of two $\mathrm{CH}_{3}$ groups. The reaction of 1-Chloro-3methylpyridobenzimidazole-4-carbonitrile 10 [7] with dimethylamine in dioxane at room temperature led to compounds 15 ; its ${ }^{1} \mathrm{HNMR}$ spectrum revealed a singlet at $2.93 \mathrm{ppm}$ representing six protons of the two $\mathrm{CH}_{3}$ groups.

\section{In vitro antitumor activity}

Nine compounds $5 b, 13 a, 13 d, 13 e, 14 b, 14 c, 15,16$, and 17 were selected by NCI and tested initially at a single dose $(10 \mu \mathrm{M})$ in the full NCI 60 cell line panel. The results are recorded as percentage growth (G\%); for example, a value of (100\%) means no growth inhibition. A value of $(20 \%)$ would mean (80\%) growth inhibition.

Results obtained for $\mathbf{5 b}$ revealed that it possesses a weak activity against CNS SNB-75 (83.72\%); renal cancer CAKI-1 (89.03\%) and UO31(79.55\%); prostate cancer PC-3 (88.18\%) and breast cancer MCF7 (86.44\%) and T-47D (86.26\%) cell lines.

The PBIs with substituted aminoethlyl moiety at position-1 (13a, 13d and 13e) showed weak activity as indicated from (G\%) for some cell lines; for compound13a: non-small cell lung cancer HOP-62 (87.18\%); melanoma SK-MEL-5 (89.05\%) and UACC-62 (81.60\%); renal cancer CAKI-1 (89.91\%) and UO-31 (80.97\%) and breast cancer MDA-MB-231/ATCC (84.44\%) and T-47D (88.55\%) cell lines. The (G\%) for PBI (13d): non-small cell lung cancer A549/80CC (83.42\%) and NCI-H522 (72.54\%); renal cancer CAKI-1 (85.61\%) and UO31 (82.36\%); and breast cancer MCF7 (88.74\%), BT-549 (87.01\%) 
Citation: Darwish SAZ, Elbayaa RY, Ashour HMA, Khalil MA, Badawey EAM (2018) Potential Anticancer Agents: Design, Synthesis of New Pyrido[1,2-a]benzimidazoles and Related Derivatives Linked to Alkylating Fragments. Med Chem (Los Angeles) 8: 86-095. doi: 10.4172/21610444.1000498

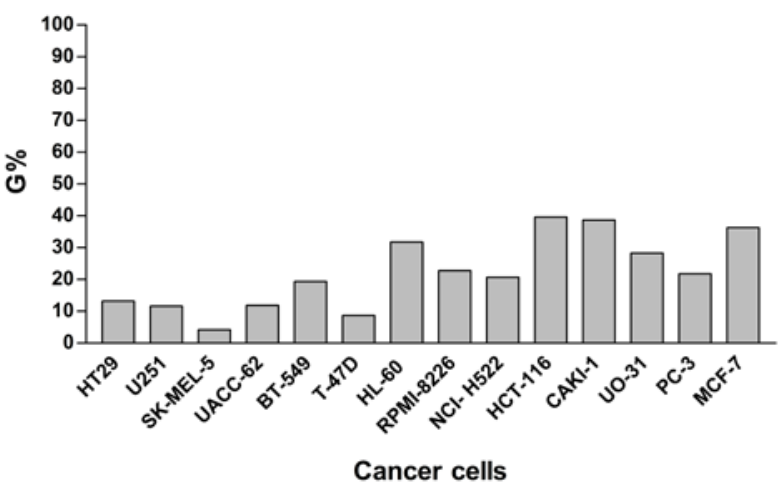

Figure 4: In vitro antitumor activity of compound $14 \mathrm{c}$ against different cancer cells.

and T-47D (68.31\%) cell lines. The $(\mathrm{G} \%)$ for related analogue (13e): non-small cell lung cancer A549/80CC (87.59\%), HOP-62 (84.71\%) and NCI-H522 (85.52\%); colon cancer HCT-116 (75.01\%) and HT29 (84.65\%); renal cancer 786-0 (86.64\%) and UO-31 (85.67\%); and breast cancer MCF7 (86.84\%), BT-549 (83.52\%) and T-47D (89.04\%) cell lines.

The PBIs with aryl substituents $(\mathbf{1 4 b}, \mathbf{c})$ showed different cytotoxic profiles. Compound $\mathbf{1 4 b}$ demonstrates a weak activity against nonsmall lung cancer A549/ATCC (72.60\%), HOP-62 (79.96\%) and NCI-H5-2 (82.00\%); melanoma UACC-62 (86.58\%); renal cancer UO31 (88.24\%); and breast cancer BT-549 (87.45\%) cell lines. However, it displayed considerable activity against leukemia HL-60(TB) (43.63\%) cell line. On the other hand significant activity was recorded for the PBI analogue (14c) against colon cancer HT-29 (13.19\%); CNS cancer U251 (11.62\%); melanoma SK-MEL-5 (4.24\%) and UACC-62 (11.90\%) and breast cancer BT- 549 (19.39\%) and T-47D (8.68\%) cell lines. In addition, it showed a remarkable activity against leukemia HL-60(TB) (31.80\%) and RPMI-8226 (22.82\%); non-small cell lung cancer NCI-H522 (20.69\%); colon cancer HCT-116 (39.61\%); renal cancer CAKI-1 (38.74\%) and UO-31 (28.32\%); prostate cancer PC-3 (21.80\%) and breast cancer MCF 7 (36.31\%) cell lines. Furthermore compound (14c) showed a considerable activity against non-small cell lung cancer A549/ATCC (43.73\%); CNS cancer SNB-19 (40.70\%) and SNB-75 (59.41\%); melanoma LOX IMVI (59.64\%); ovarian cancer SKOV-3 (46.41\%); renal cancer 786-0 (58.95\%) and breast cancer MDAMB-231/ATCC (54.18\%) cell lines (Figure 4).

The PBIs with dimethylamino substituent (15) showed weak cytotoxic activity against some cell lines as indicated from the recorded (G\%): non-small cell lung cancer A549/ATCC (66.70\%), HOP-62 (80.34\%) and NCI-H522 (69.49\%); renal cancer 786-0 (80.56\%), CAKI$1(82.60 \%)$ and UO-31 (77.15\%) and breast 80 cancer BT-549 (77.67\%) and T-47D (65.75\%) cell lines. The PBI (16) was completely inactive; whereas, its chloro analogue (17) displayed remarkable activity against colon cancer HCT-116(22.15\%) cell line. It also showed weak inhibitory effect against leukemia HL-60 (TB) (85.53\%) and RPMI-226 (85.36\%); non-small cell lung cancer NCI-H522 (83.88\%); colon cancer HT29 (86.43\%); melanoma LOXIMVI (84.85\%) and UACC-62 (76.98\%); renal cancer $786-0(83.66 \%)$ and UO-31 (81.23\%); prostate cancer PC-3 (88.87\%) and breast cancer MCF-7 (77.13\%), MDA-MB-231/ ATCC (81.11\%) and T-47D (82.20\%) cell lines.

\section{Structure Activity Relationship (SAR)}

Compound $\mathbf{5 b}$ revealed a weak inhibitory effect against many cell lines from some types of cancer. This would indicate that the presence of 2-pyridone scaffold did not result in a significant improvement on the activity. Structural activity correlation revealed that the PBIs which lack the 2-pyridone unit but have instead a pyridine moiety showed variable cytotoxic activity. For instance, substitution at position-1 with $\mathrm{N}, \mathrm{N}$-dimethylaminoethylamino (13a), morpholinoethylamino (13d) and piperazinylethylamino (13e) did not let significant activity. On the other hand, the PBI which carry 4-methoxyphenylamino moiety (14b) exhibited weak inhibitory effects against several cell lines from non-small cell lung cancer, melanoma, renal and breast cancer and it demonstrated remarkable cytotoxic activity against one cell line from leukemia. It is worthy to mention that the highest anticancer activity was recorded for compounds 14c. Results revealed that replacement of the 4-methoxy (14b) with 3-trifluoromethyl group (14c) resulted in broad spectrum and variable degree of activity against many of the tested cell lines. In fact, this finding would indicate the impact of the lipophilic 3-trifluoromethylphenylamino substituents on the activity.

The presence of bis(2-hydroxyethyl)amino group (16) did not show any significant impact on the activity; whereas, the PBI (17) which carry an alkylating fragment, bis(2-choloroethyl)amino, displayed a remarkable inhibitory effect against colon cancer HCT-116 cell line and weak inhibitory effects against many cell lines from leukemia, melanoma, non-small lung cancer and colon, renal, prostate and breast cancer.

\section{Conclusion}

In conclusion, two series of benzimidazoles comprising pyridine and 2-pyridone nuclei together with various functionalities believed to have alkylation ability were synthesized. These series were designed as an example of a new molecular hybrids having anticancer activity. The anticancer activity results revealed that among the tested compounds, compound (14c) was found to possess promising anticancer activity and the most significant inhibition as revealed from the growth percentage (G\%)was found against melanoma SK-MEL-5 (4.24\%) and UACC-62 (11.90\%), CNS cancer U251 (11.62\%), colon cancer HT-29 (13.19\%); and breast cancer BT- 549 (19.39\%) and T-47D (8.68\%) cell lines. In fact, this finding together with the remarkable antineoplastic activity reported to the related PBI analogues (NSC682011 and NSC699944, Figure 1) would indicate the impact of the lipophilic 3-tripfluoromethylphenylamino and 4-fluorophenylamino substituents on the activity. Although, none of the screened compounds satisfied the threshold inhibition criteria to pass for evaluation in the full panel fivedose in vitro antitumor screen, The PBI $\mathbf{1 4 c}$ can be considered starting structure that merit further optimization in order to design more active lead compounds for further studies.

\section{Declaration of Interest}

The authors declared no conflicts of interest. Only the authors are responsible for the content and writing of the paper.

\section{References}

1. Fujisawa D, Inoguchi H, Shimoda H, Yoshiuchi K, Inoue S, et al. (2016) Impact of depression on health utility value in cancer patients. Psychooncology 25: 491-495.

2. Mathew B, Hobrath JV, Connelly MC, Kiplin Guy R, Reynolds RC (2017) Diverse amide analogs of sulindac for cancer treatment and prevention. Bioorg Med Chem Lett 15 27: 4614-4621.

3. Badawey EAM, Kappe T (1995) Benzimidazole condensed ring system. IX Potential antineoplastics. New synthesis of some pyrido [1, 2- $\alpha]$ benzimidazoles and related derivative. Eur J Med Chem 30: 327-332. 
Citation: Darwish SAZ, Elbayaa RY, Ashour HMA, Khalil MA, Badawey EAM (2018) Potential Anticancer Agents: Design, Synthesis of New Pyrido[1,2-a]benzimidazoles and Related Derivatives Linked to Alkylating Fragments. Med Chem (Los Angeles) 8: 86-095. doi: 10.4172/21610444.1000498

4. Badawey EAM, Kappe T (1999) Benzimidazole condensed ring systems. XI. Synthesis of some substituted cycloalkyl pyrido [1,2-a] benzimidazoles with anticipated antineoplastic activity. Eur J Med Chem 34: 663-667.

5. El-Hawash SAM, Badawey EAM, Kappe T (1999) Benzimidazole condensed ring systems. XII. Synthesis and anticancer evaluation of certain pyrido [1, 2-a] benzimidazole derivatives. Pharmazie 54: 341-346.

6. Badawey EAM, Rida SM, Soliman FSG, Kappe T (1989) Benzimidazole condensed ring systems. III. Synthesis of some substituted 2,3-dihydrocyclopenta-1 $\mathrm{H}-\left[4^{\prime}, 5^{\prime}: 2,3\right]$ pyrido[1,2-a]benzimidazole-11carbonitriles. Monatsh Chem 120: 73-76.

7. Rida SM, Soliman FSG, Badawey EAM, Kappe T (1988) Benzimidazole condensed ring systems. 2. New synthesis of substituted $1 \square$ oxo $\square 1 \mathrm{H}, 5 \mathrm{H}$-pyrido [1, 2-a] benzimidazole-4-carbonitriles and related derivatives. J Heterocyclic Chem 25: 1725-1728.

8. Badawey EAM, Kappe T (1995) Benzimidazole condensed ring systems 10 (1). Synthesis and Cytotoxic activity of some pyrido [1, 2-a] benzimidazoles. IL Farmaco 50: 537-542.

9. Baumann M, Baxendale IR (2013) An overview of the synthetic routes to the best-selling drugs containing 6-membered heterocycles. Beilstein J Org Chem 9: 2265-2319.

10. Sweetman SC (2009) Martindale. The Complete Drug Reference. 36th edn Pharmaceutical Press, pp: 117-177.

11. Parlow JJ, Kurumbail RG, Stegeman RA, Stevens AM, Stallings WC, et al. (2003) Design, synthesis, and crystal structure of selective 2-pyridone tissue factor VIla inhibitors. J Med Chem 46: 4696-4701.

12. Sweetman SC (2009) Martindale. The Complete Drug Reference. 36th edn. Pharmaceutical Press, pp: 882-884.

13. Desai NC, Shihory NR, Kotadiya GM (2014) Facile synthesis of benzimidazole bearing 2-pyridone derivatives as potential antimicrobial agents. Chinese Chemical Letters 25: 305-307.

14. Gupta AK, Plott T (2004) Ciclopirox: a broad $\square$ spectrum antifungal with antibacterial and anti $\square$ inflammatory properties. International Journal of Dermatology 43: 3-8.

15. Hamilton G, Olszewski U (2013) Picoplatin pharmacokinetics and chemotherapy of non-small cell lung cancer. Expert Opin Drug Metab Toxicol 9: 1381-1390.

16. Sweetman SC (2009) Martindale. The Complete Drug Reference. 36th edn.
Pharmaceutical Press, pp: 733-770.

17. Isaacs JT (2010) The long and winding road for the development of tasquinimod as an oral second-generation quinoline-3-carboxamide antiangiogenic drug for the treatment of prostate cancer. Expert Opin Investig Drugs 19: 1235-1243.

18. Maskey RP, Grun-Wollny I, Laatsch H (2005) Isolation and structure elucidation of Diazaquinomycin $\mathrm{C}$ from a terrestrial streptomyces $\mathrm{sp}$. and confirmation of the akashin structure. Natural Product Research 19: 137-142.

19. Jimenez J (2008) Cytotoxicity of the $\beta$-carboline alkaloids harmine and harmaline in human cell assays in vitro. Experimental and Toxicologic Pathology 60: 381-389.

20. Thomas CJ, Rahier NJ, Hecht SM (2004) Camptothecin: current perspectives. Bioorganic \& Medicinal Chemistry 12: 1585-1604.

21. Staker BL, Feese MD, Cushman M (2005) Structures of three classes of anticancer agents bound to the human topoisomerase I- DNA covalent complex. J Med Chem 48: 2336-2345.

22. Dogra S, Awasthi P, Tripathi S, Pradeep TP, Nair MS, et al. (2014) NMR-based structure of anticancer drug mitoxantrone stacked with terminal base pair of DNA hexamer sequence d-(ATCGAT)2. J Biomol Struct Dyn 32: 1164-1183.

23. Tanis JB, Mason SL, Maddox TW, Blackwood L, Killick DR, et al. (2018) Evaluation of a multi-agent chemotherapy protocol combining lomustine procarbazine and prednisolone (LPP) for the treatment of relapsed canine nonHodgkin high-grade lymphomas. Vet Comp Oncol.

24. Keating MJ, Bach C, Yasothan U, Kirkpatrick P (2008) Bendamustine. Nat Rev Drug Discov 7: 473-474.

25. Sweetman SC (2009) Martindale. The Complete Drug Reference. 36th edn Pharmaceutical Press, pp: 754-755.

26. Alley MC, Scudiere DA, Monks A, Hursey ML, Czerwinski MJ, et al. (1988) Feasibility of drug screening with panels of human tumor cell lines using a microculture tetrazolium assay. Cancer Research 48: 589-601.

27. Boyd MR, Paull KD (1995) Some practical considerations and applications of the National Cancer Institute in vitro anticancer drug discovery screen. Drug Development Research 34: 91-109.

28. Grever MR, Schepartz SA, Chabner BA (1992) The National Cancer Institute: Cancer drug discovery and development program. Semin Oncol 19: 622-638.

29. Shoemaker $\mathrm{RH}$ (2006) The $\mathrm{NCl} 60$ human tumour cell line anticancer drug screen. Nature Reviews 6: 813-823. 